\title{
The Modular Degree, Congruence Primes, and Multiplicity One
}

\author{
Amod Agashe ${ }^{1}$ Kenneth A. Ribet William A. Stein
}

\begin{abstract}
.
The modular degree and congruence number are two fundamental invariants of an elliptic curve over the rational field. Frey and Müller have asked whether these invariants coincide. We find that the question has a negative answer, and show that in the counterexamples, multiplicity one (defined below) does not hold. At the same time, we prove a theorem about the relation between the two invariants: the modular degree divides the congruence number, and the ratio is divisible only by primes whose squares divide the conductor of the elliptic curve. We discuss the ratio even in the case where the square of a prime does divide the conductor, and we study analogues of the two invariants for modular abelian varieties of arbitrary dimension.
\end{abstract}

\section{Introduction}

Let $E$ be an elliptic curve over $\mathbf{Q}$. By [BCDT01], we may view $E$ as an abelian variety quotient over $\mathbf{Q}$ of the modular Jacobian $J_{0}(N)$, where $N$ is the conductor of $E$. We assume that the kernel of the map $J_{0}(N) \rightarrow E$ is connected, i.e., that $E$ is an optimal quotient of $J_{0}(N)$ (this can always be done by replacing $E$ by an isogenous curve if needed). The modular degree $m_{E}$ is the degree of the composite map $X_{0}(N) \rightarrow J_{0}(N) \rightarrow E$, where we map $X_{0}(N)$ to $J_{0}(N)$ by sending $P \in X_{0}(N)$ to $[P]-[\infty] \in J_{0}(N)$.

Let $f_{E}=\sum a_{n} q^{n} \in S_{2}\left(\Gamma_{0}(N), \mathbf{C}\right)$ be the newform attached to $E$. The congruence number $r_{E}$ of $E$ is the largest integer such that there is an element $g=\sum b_{n} q^{n} \in S_{2}\left(\Gamma_{0}(N)\right)$ with integer Fourier coefficients $b_{n}$ that is orthogonal to $f_{E}$ with respect to the Petersson inner product, and congruent to $f_{E}$ modulo $r_{E}$ (i.e., $a_{n} \equiv b_{n}\left(\bmod r_{E}\right)$ for all $\left.n\right)$.

\footnotetext{
${ }^{1}$ Agashe was partially supported by National Science Foundation Grant No. 0603668. Stein was partially supported by National Science Foundation Grant No. 0653968.
} 
Section 2 is about relations between $r_{E}$ and $m_{E}$. For example, $m_{E} \mid r_{E}$. In [FM99, Q. 4.4], Frey and Müller asked whether $r_{E}=m_{E}$. We give examples in which $r_{E} \neq m_{E}$, and show that in these examples, there is a maximal ideal $\mathfrak{m}$ of the Hecke algebra $\mathbf{T}$, such that $J_{0}(N)[\mathfrak{m}]$ has dimension more than two over $\mathbf{T} / \mathfrak{m}$ (this is the failure of multiplicity one alluded to above). We then conjecture that for any prime $p, \operatorname{ord}_{p}\left(r_{E} / m_{E}\right) \leq \frac{1}{2} \operatorname{ord}_{p}(N)$, and prove this conjecture when $\operatorname{ord}_{p}(N) \leq 1$.

In Section 3, we consider analogs of the modular degree and the congruence number for certain modular abelian varieties that are not necessarily elliptic curves; these include optimal quotients of $J_{0}(N)$ and $J_{1}(N)$ of any dimension associated to newforms. Section 3 may be read independently of Section 2 . In Sections 4 and 5 we prove the main theorem of this paper (Theorem 3.6), and also give some examples of failure of what we call multiplicity one for differentials (see Definition 5.13).

Acknowledgment. The authors are grateful to M. Baker, F. Calegari, B. Conrad, J. Cremona, G. Frey, H.W. Lenstra, and B. Noohi for discussions and advice regarding this paper. We would especially like to thank B. Conrad for the material in the appendix and for his suggestions concerning a number of technical facts that are inputs to our arguments. The first author is also grateful to the Max-Planck-Institut für Mathematik for their hospitality during a visit when he partly worked on this paper.

\section{Elliptic CURVES}

In Section 2.1, we discuss relationships between the modular degree and the congruence number of an elliptic curve. In Section 2.2 we recall the notion of multiplicity one and give new examples in which it fails.

\subsection{MODUlar DEGREE AND CONGRUENCE NUMBER}

Let $N$ be a positive integer and let $X_{0}(N)$ be the modular curve over $\mathbf{Q}$ that classifies isomorphism classes of elliptic curves with a cyclic subgroup of order $N$. The Hecke algebra $\mathbf{T}$ of level $N$ is the subring of the ring of endomorphisms of $J_{0}(N)=\operatorname{Jac}\left(X_{0}(N)\right)$ generated by the Hecke operators $T_{n}$ for all $n \geq 1$. Let $f$ be a newform of weight 2 for $\Gamma_{0}(N)$ with integer Fourier coefficients, and let $I_{f}$ be kernel of the homomorphism $\mathbf{T} \rightarrow \mathbf{Z}\left[\ldots, a_{n}(f), \ldots\right]$ that sends $T_{n}$ to $a_{n}$. Then the quotient $E=J_{0}(N) / I_{f} J_{0}(N)$ is an elliptic curve over $\mathbf{Q}$. We call $E$ the optimal quotient associated to $f$. Composing the embed$\operatorname{ding} X_{0}(N) \hookrightarrow J_{0}(N)$ that sends $\infty$ to 0 with the quotient map $J_{0}(N) \rightarrow E$, we obtain a surjective morphism of curves $\phi_{E}: X_{0}(N) \rightarrow E$. Recall that the modular degree $m_{E}$ of $E$ is the degree of $\phi_{E}$.

Let $S_{2}(\mathbf{Z})$ denote the group of cuspforms of weight 2 on $\Gamma_{0}(N)$ with integral Fourier coefficients, and if $G$ is a subgroup of $S_{2}(\mathbf{Z})$, let $G^{\perp}$ denote the subgroup of $S_{2}(\mathbf{Z})$ consisting of cuspforms that are orthogonal to $f$ with respect to the Petersson inner product. The congruence number of $E$ (really, that of $f$ ) is the 
positive integer $r_{E}$ defined by any of the following equivalent conditions:

(i) $r_{E}$ is the largest integer $r$ such that there exists $g \in(\mathbf{Z} f)^{\perp}$ with $f \equiv g \bmod r$.

(ii) $r_{E}$ is the order of the quotient group $\frac{S_{2}(\mathbf{Z})}{\mathbf{Z} f+(\mathbf{Z} f)^{\perp}}$.

We say that a prime is a congruence prime for $E$ if it divides the congruence number $r_{E}$. Congruence primes have been studied by Doi, Hida, Ribet, Mazur and others (see, e.g., [Rib83, §1]), and played an important role in Wiles's work [Wi195] on Fermat's last theorem. Frey and Mai-Murty have observed that an appropriate asymptotic bound on the modular degree is equivalent to the $a b c$-conjecture (see [Fre97, p.544] and [Mur99, p.180]). Thus, results that relate congruence primes and the modular degree may be of great interest.

TheOREM 2.1. Let $E$ be an elliptic curve over $\mathbf{Q}$ of conductor $N$, with modular degree $m_{E}$ and congruence number $r_{E}$. Then $m_{E} \mid r_{E}$ and if $\operatorname{ord}_{p}(N) \leq 1$ then $\operatorname{ord}_{p}\left(r_{E}\right)=\operatorname{ord}_{p}\left(m_{E}\right)$.

Thus any prime that divides the modular degree of an elliptic curve $E$ is a congruence prime for $E$, and if $p$ is a congruence prime for $E$ such that $p^{2}$ does not divide the conductor of $E$, then $p$ divides the modular degree of $E$. The divisibility $m_{E} \mid r_{E}$ was first discussed in [Zag85, Th. 3], where it is attributed to the second author (Ribet); however in [Zag85] the divisibility was mistakenly written in the opposite direction. For some other expositions of the proof that $m_{E} \mid r_{E}$, see [AU96, Lem 3.2] and [CK04]. We generalize this divisibility and prove it in Theorem 3.6(a). The second part of Theorem 2.1, i.e., that if $\operatorname{ord}_{p}(N) \leq 1$ then $\operatorname{ord}_{p}\left(r_{E}\right)=\operatorname{ord}_{p}\left(m_{E}\right)$, follows from the more general Theorem 3.6(b) below. Note that [AU96, Prop. 3.3-3.4] implies the weaker statement that if $p \nmid N$, then $\operatorname{ord}_{p}\left(r_{E}\right)=\operatorname{ord}_{p}\left(m_{E}\right)$, since [AU96, Prop. 3.3] implies

$$
\operatorname{ord}_{p}\left(r_{E}\right)-\operatorname{ord}_{p}\left(m_{E}\right)=\operatorname{ord}_{p}(\# \mathcal{C})-\operatorname{ord}_{p}\left(c_{E}\right)-\operatorname{ord}_{p}(\# \mathcal{D}),
$$

and by [AU96, Prop. 3.4], $\operatorname{ord}_{p}(\# \mathcal{C})=0$. Here $c_{E}$ is the Manin constant of $E$, which is an integer (e.g., see [ARS06]), and $\mathcal{C}$ and $\mathcal{D}$ are certain groups.

Frey and Müller [FM99, Ques. 4.4] asked whether $r_{E}=m_{E}$ in general. After implementing an algorithm to compute $r_{E}$ in Magma [BCP97], we quickly found that the answer is no. The counterexamples at conductor $N \leq 144$ are given in Table 1, where the curve is given using the notation of [Cre97].

For example, the elliptic curve 54B1, given by the equation $y^{2}+x y+y=$ $x^{3}-x^{2}+x-1$, has $r_{E}=6$ and $m_{E}=2$. To see explicitly that $3 \mid r_{E}$, observe that the newform corresponding to $E$ is $f=q+q^{2}+q^{4}-3 q^{5}-q^{7}+\cdots$ and the newform corresponding to $X_{0}(27)$ is $g=q-2 q^{4}-q^{7}+\cdots$, so $g(q)+g\left(q^{2}\right)$ appears to be congruent to $f$ modulo 3 . To prove this congruence, we checked it for 18 Fourier coefficients, where the sufficiency of precision to degree 18 was determined using [Stu87].

It is unclear whether there is a bound on the possible primes $p$ that occur. For example, for the curve 242B1 of conductor $N=2 \cdot 11^{2}$ we have

$$
m_{E}=2^{4} \neq r_{E}=2^{4} \cdot 11 .
$$


Table 1: Differing Modular Degree and Congruence Number

\begin{tabular}{|l|l|l|}
\hline Curve & $m_{E}$ & $r_{E}$ \\
\hline $54 \mathrm{~B} 1$ & 2 & 6 \\
\hline $64 \mathrm{~A} 1$ & 2 & 4 \\
\hline $72 \mathrm{~A} 1$ & 4 & 8 \\
\hline 80A1 & 4 & 8 \\
\hline $88 \mathrm{~A} 1$ & 8 & 16 \\
\hline $92 \mathrm{~B} 1$ & 6 & 12 \\
\hline $96 \mathrm{~A} 1$ & 4 & 8 \\
\hline $96 \mathrm{~B} 1$ & 4 & 8 \\
\hline
\end{tabular}

\begin{tabular}{|l|l|l|}
\hline Curve & $m_{E}$ & $r_{E}$ \\
\hline 99A1 & 4 & 12 \\
\hline 108A1 & 6 & 18 \\
\hline 112A1 & 8 & 16 \\
\hline 112B1 & 4 & 8 \\
\hline 112C1 & 8 & 16 \\
\hline 120A1 & 8 & 16 \\
\hline 124A1 & 6 & 12 \\
\hline 126A1 & 8 & 24 \\
\hline
\end{tabular}

\begin{tabular}{|l|l|l|}
\hline Curve & $m_{E}$ & $r_{E}$ \\
\hline $128 \mathrm{~A} 1$ & 4 & 32 \\
\hline $128 \mathrm{~B} 1$ & 8 & 32 \\
\hline $128 \mathrm{C} 1$ & 4 & 32 \\
\hline $128 \mathrm{D} 1$ & 8 & 32 \\
\hline $135 \mathrm{~A} 1$ & 12 & 36 \\
\hline $144 \mathrm{~A} 1$ & 4 & 8 \\
\hline $144 \mathrm{~B} 1$ & 8 & 16 \\
\hline & & \\
\hline
\end{tabular}

We propose the following replacement for Question 4.4 of [FM99]:

ConjeCture 2.2. Let $E$ be an optimal elliptic curve of conductor $N$ and $p$ be any prime. Then

$$
\operatorname{ord}_{p}\left(\frac{r_{E}}{m_{E}}\right) \leq \frac{1}{2} \operatorname{ord}_{p}(N)
$$

We verified Conjecture 2.2 using Sage $\left[\mathrm{S}^{+} 09\right]$ for every optimal elliptic curve quotient of $J_{0}(N)$, with $N \leq 557$.

If $p \geq 5$ then $\operatorname{ord}_{p}(N) \leq 2$, so a special case of the conjecture is

$$
\operatorname{ord}_{p}\left(\frac{r_{E}}{m_{E}}\right) \leq 1 \quad \text { for any } p \geq 5 .
$$

\subsection{Multiplicity one AND its failure}

We say that a maximal ideal $\mathfrak{m}$ of $\mathbf{T}$ satisfies multiplicity one if $J_{0}(N)[\mathfrak{m}]$ is of dimension two over $\mathbf{T} / \mathfrak{m}$. The reason one calls this "multiplicitly one" is that if the canonical two dimensional representation $\rho_{\mathfrak{m}}$ over $\mathbf{T} / \mathfrak{m}$ attached to $\mathfrak{m}$ (e.g., see [Rib90, Prop. 5.1]) is irreducible, then $J_{0}(N)[\mathfrak{m}]$ is a direct sum of copies of $\rho_{\mathfrak{m}}$ [Rib90, Thm. 5.2], and a maximal ideal $\mathfrak{m}$ of $\mathbf{T}$ satisfies multiplicity one precisely if the multiplicity of $\rho_{\mathfrak{m}}$ in this decomposition is one. Even if $\rho_{\mathfrak{m}}$ is reducible, the definition of multiplicity one given above is relevant (e.g., see [Maz77, Cor. 16.3]). The notion of multiplicity one, originally found in Mazur [Maz77], has played an important role in several places (e.g., in Wiles's proof of Fermat's last theorem: see Thm. 2.1 in [Wil95]).

In [MR91, §13], the authors find examples of failure of multiplicity one in which if $p$ is the residue characteristic of $\mathfrak{m}$, then $p^{3} \mid N$, and $\rho_{\mathfrak{m}}$ is modular of level $N / p^{2}$. Kilford [Kil02] found examples of failure of multiplicity one where $N$ is prime and the residue characteristic of $\mathfrak{m}$ is 2. See also [Wie07] and [KW08] for examples of failure of multiplicity one in the $\Gamma_{1}(N)$ context. We now give examples of failure of multiplicity one where the square of the 
residue characteristic of $\mathfrak{m}$ divides the level (the residue characteristic is often odd).

Proposition 2.3. Suppose $E$ is an optimal elliptic curve over $\mathbf{Q}$ of conductor $N$ and $p$ is a prime such that $p \mid r_{E}$ but $p \nmid m_{E}$. Then there is a maximal ideal $\mathfrak{m}$ of $\mathbf{T}$ with residue characteristic p such that $\operatorname{dim}_{\mathbf{T} / \mathfrak{m}} J_{0}(N)[\mathfrak{m}]>2$, i.e., multiplicity one fails for $\mathfrak{m}$.

The proposition follows from the more general Proposition 5.9 below. It follows from the proposition above that any example in Table 1 where simultaneously a prime divides $r_{E}$ but does not divide $m_{E}$ provides an example of failure of multiplicity one. In such examples, the associated representation $\rho_{\mathfrak{m}}$ may or may not be irreducible. For example, for the elliptic curve 54B1 and $p=3$, we have $\operatorname{ord}_{3}\left(r_{E}\right)=1$ but $\operatorname{ord}_{3}\left(m_{E}\right)=0$, so there is a maximal ideal $\mathfrak{m}$ with residue characteristic 3 such that multiplicity one fails for $\mathfrak{m}$. The curve 54B1 has rational 3-torsion, so $\rho_{\mathfrak{m}}$ is reducible. On the other hand, for the elliptic curve 99A1, we have $\operatorname{ord}_{3}\left(r_{E}\right)=1$ but $\operatorname{ord}_{3}\left(m_{E}\right)=0$, so again there is a maximal ideal $\mathfrak{m}$ with residue characteristic 3 such that multiplicity one fails for $\mathfrak{m}$. Moreover, $J_{0}(99)$ is isogenous to a product of elliptic curves, none of which admit a rational 3-isogeny. Hence $\rho_{\mathfrak{m}}$ is irreducible.

The notion of multiplicity one at a maximal ideal $\mathfrak{m}$ is closely related to Gorensteinness of the completion of $\mathbf{T}$ at $\mathfrak{m}$ (e.g., see [Til97]). Kilford [Kil02] found examples of failure of Gorensteinness (and multiplicity one) at the prime 2 for certain prime levels. In the examples as above where multiplicity one fails for some maximal ideal, it would be interesting to do computations (e.g., as in [Kil02]) to see if the completion of the Hecke algebra at the maximal ideal is Gorenstein or not.

\section{MOdUlar ABELIAN VARIETIES OF ARBITRARY DIMENSION}

For $N \geq 4$, let $\Gamma$ be either $\Gamma_{0}(N)$ or $\Gamma_{1}(N)$. Let $X$ be the modular curve over $\mathbf{Q}$ associated to $\Gamma$, and let $J$ be the Jacobian of $X$. Let $A$ and $B$ be abelian subvarieties of $J$ such that $A+B=J, A \cap B$ is finite, and every endomorphism of $J$ over $\mathbf{Q}$ preserves $A$ and $B$. In this section, we generalize the notions of the congruence number and the modular degree to subvarieties $A$ as above, and state a theorem relating the two numbers, which we prove in Sections 4 and 5.

We first give a general example of $A$ and $B$ as above. Up to isogeny, $J$ is the product of factors $J_{f}^{e(f)}$ where $f$ runs over the set of newforms of level dividing $N$, taken up to Galois conjugation, and $e(f)$ is the number of divisors of $N / N(f)$, where $N(f)$ is the level of $f$. Here $J_{f}$ is the standard abelian subvariety of $J$ attached to $f$ by Shimura [Shi94, Thm. 7.14]. Let $A^{\prime}$ be the sum of $J_{f}^{e(f)}$ for some set of $f$ 's (taken up to Galois conjugation), and let $B^{\prime}$ be the sum of all the other $J_{f}^{e(f)}$ 's. Clearly $A^{\prime}+B^{\prime}=J$. The $J_{f}$ 's are simple (over $\mathbf{Q}$ ), hence $A^{\prime} \cap B^{\prime}$ is finite. In view of the following lemma, $A^{\prime}$ and $B^{\prime}$ 
provide an example of $A$ and $B$ respectively as above. Note that by $\operatorname{End}(J)$ we mean the ring of endomorphisms of $J$ defined over $\mathbf{Q}$.

Lemma 3.1. $\operatorname{End}(J)$ preserves $A^{\prime}$ and $B^{\prime}$.

Proof. Suppose $\operatorname{End}(J)$ does not preserve $A^{\prime}$ (the case of $B^{\prime}$ is symmetric). Then since the $J_{f}$ 's are simple, that means that some abelian subvariety $J_{g}$ of $A^{\prime}$ is isogenous to some abelian subvariety $J_{h}$ of $B^{\prime}$, where $g \neq h$. Pick a prime $\ell$. If $f$ is a newform, then let $\rho_{f}$ denote the canonical absolutely irreducible $\ell$-adic representation attached to $f$. Now $\overline{\mathbf{Q}}_{\ell} \otimes V_{\ell}\left(J_{f}\right)^{\mathrm{ss}}$ is a direct sum of copies of $\rho_{\sigma(f)}$ as $\sigma$ ranges over all embeddings into $\overline{\mathbf{Q}}$ of the field generated by the Fourier coefficients of $f$. Thus the above implies that there are distinct newforms $g^{\prime}$ and $h^{\prime}$ (of some level dividing $N$ ) such that $\rho_{g^{\prime}} \cong \rho_{h^{\prime}}$. Now each $\rho_{f}$ satisfies $\operatorname{tr}\left(\rho_{f}\left(\operatorname{Frob}_{p}\right)\right)=a_{p}(f)$ for all $p \nmid N \ell$. Thus for all $p \nmid N \ell$, we have $a_{p}\left(g^{\prime}\right)=a_{p}\left(h^{\prime}\right)$. By the multiplicity one theory (e.g., see [Li75, Cor. 3, pg. 300]), this means that $g^{\prime}=h^{\prime}$, a contradiction.

We now give a more specific example, which will include the case of elliptic curves. Recall that $\mathbf{T}$ denotes the Hecke algebra. If $f=\sum a_{n}(f) q^{n} \in S_{2}(\Gamma)$ is a newform and $I_{f}=\operatorname{ker}\left(\mathbf{T} \rightarrow \mathbf{Z}\left[\ldots, a_{n}(f), \ldots\right]\right)$, then $A_{f}=J / I_{f} J$ is the newform quotient associated to $f$. It is an abelian variety over $\mathbf{Q}$ of dimension equal to the degree of the field $\mathbf{Q}\left(\ldots, a_{n}(f), \ldots\right)$. Let $\phi_{2}$ denote the quotient map $J \rightarrow A$. If $C$ is an abelian variety, then we denote its dual abelian variety by $C^{\vee}$. There is a canonical principal polarization $\theta: J \cong J^{\vee}$. Dualizing $\phi_{2}$, we obtain a closed immersion $\phi_{2}^{\vee}: A_{f}^{\vee} \rightarrow J^{\vee}$, which when composed with $\theta^{-1}$ : $J^{\vee} \cong J$ gives us an injection $\phi_{1}: A_{f}^{\vee} \hookrightarrow J$. One slight complication is that the isomorphim $\theta$ does not respect the action of $\mathbf{T}$, because if $T$ is a Hecke operator on $J$, then on $J^{\vee}$ it acts as $W_{N} T W_{N}$, where $W_{N}$ is the Atkin-Lehner involution (see e.g., [DI95, Rem. 10.2.2]). However, on the new quotient $J^{\text {new }}$, the action of the Hecke operators commutes with that of $W_{N}$, so since the quotient map $J \rightarrow$ $A_{f}$ factors through $J^{\text {new }}$, the Hecke action on $A_{f}^{\vee}$ induced by the embedding $A_{f}^{\vee} \rightarrow J^{\vee}$ and the action on $A_{f}^{\vee}$ induced by the injection $\phi_{1}: A_{f}^{\vee} \rightarrow J$ are the same. Hence $A_{f}^{\vee}$ is isomorphic to $\phi_{1}\left(A_{f}^{\vee}\right)$ as a T-module, and $\phi_{1}\left(A_{f}^{\vee}\right)=J_{f}$ (this follows from the characterization of $J_{f}$ in [Shi94, Thm. 7.14]). For simplicity, we will often denote $\phi_{1}\left(A_{f}^{\vee}\right)=J_{f}$ by just $A_{f}^{\vee}$. Let $\phi$ be the composite map $A_{f}^{\vee} \stackrel{\phi_{1}}{\longrightarrow} J \stackrel{\phi_{2}}{\longrightarrow} A_{f}$; then $\phi$ is a polarization (induced by dual of the polarization of $J$ ). Thus $A_{f}^{\vee}+I_{f} J=J$ and $A_{f}^{\vee} \cap I_{f} J$ is finite. Hence, in view of Lemma 3.1, $A_{f}^{\vee}$ and $I_{f} J$ provide an example of $A$ and $B$ as in the beginning of this section.

The exponent of a finite group $G$ is the smallest positive integer $n$ such that every element of $G$ has order dividing $n$ (i.e., such that for all $x \in G, n x=0$ ).

Definition 3.2. The modular exponent $\tilde{n}_{A}$ of $A$ is the exponent of $A \cap B$ and the modular number $n_{A}$ of $A$ is its order.

Note that the definition is symmetric with respect to $A$ and $B$. In fact, the definition depends on both $A$ and $B$, unlike what the notation may suggest-we 
have suppressed the dependence on $B$ for ease of notation, with the understanding that there is a natural choice of $B$ (e.g., this is the case in the examples we gave above). If $f$ is a newform, then by the modular exponent/number of $A_{f}$, we mean that of $A=A_{f}^{\vee}$, with $B=I_{f} J$. In this situation, since $\phi$ is a polarization, $n_{A_{f}}$ is a perfect square (e.g., see [AS05, Lemma 3.14]). When $A_{f}$ is an elliptic curve, $\phi$ is multiplication by the modular degree $m_{E}$. Hence $A \cap B=\operatorname{ker}(\phi)$ is $\left(\mathbf{Z} / m_{E} \mathbf{Z}\right)^{2}$, and so for elliptic curves, the modular exponent is equal to the modular degree and the modular number is the square of the modular degree.

If $R$ is a subring of $\mathbf{C}$, let $S_{2}(R)=S_{2}(\Gamma ; R)$ denote the subgroup of $S_{2}(\Gamma ; \mathbf{C})$ consisting of cups forms whose Fourier expansions at the cusp $\infty$ have coefficients in $R$. There is a $\mathbf{T}$-equivariant bilinear pairing $\mathbf{T} \times S_{2}(\mathbf{Z}) \rightarrow \mathbf{Z}$ given by $(t, g) \mapsto a_{1}(t(g))$, which is perfect by [AU96, Lemma 2.1] (see also [Rib83, Theorem 2.2]). The action of $\mathbf{T}$ on $\mathrm{H}_{1}(J, \mathbf{Z})$ is a faithful representation that embeds $\mathbf{T}$ into $\mathrm{Mat}_{2 d}(\mathbf{Z}) \cong \mathbf{Z}^{(2 d)^{2}}$. But $\mathbf{Z}$ is Noetherian, so $\mathbf{T}$ is finitely generated over $\mathbf{Z}$, and hence so is $S_{2}(\mathbf{Z})$. Let $\mathbf{T}_{A}$ be the image of $\mathbf{T}$ in $\operatorname{End}(A)$, and let $\mathbf{T}_{B}$ be the image of $\mathbf{T}$ in $\operatorname{End}(B)$ (since $\mathbf{T} \subset \operatorname{End}(J)$, $\mathbf{T}$ preserves $A$ and $B$ ). Since $A+B=J$, the natural map $\mathbf{T} \rightarrow \mathbf{T}_{A} \oplus \mathbf{T}_{B}$ is injective, and moreover, its cokernel is finite (since $A \cap B$ is finite).

Let $S_{A}=\operatorname{Hom}\left(\mathbf{T}_{A}, \mathbf{Z}\right)$ and $S_{B}=\operatorname{Hom}\left(\mathbf{T}_{B}, \mathbf{Z}\right)$ be subgroups of $S_{2}(\mathbf{Z})$ obtained via the pairing above. Let $\operatorname{Ext}^{1}=$ Ext $_{\mathbf{Z}}^{1}$ denote the first Ext functor in the category of $\mathbf{Z}$-modules.

Lemma 3.3. There is a canonical isomorphism of $\mathbf{T}$-modules

$$
\operatorname{Ext}^{1}\left(\left(\mathbf{T}_{A} \oplus \mathbf{T}_{B}\right) / \mathbf{T}, \mathbf{Z}\right) \cong S_{2}(\mathbf{Z}) /\left(S_{A}+S_{B}\right) .
$$

The groups $S_{2}(\mathbf{Z}) /\left(S_{A}+S_{B}\right)$ and $\left(\mathbf{T}_{A} \oplus \mathbf{T}_{B}\right) / \mathbf{T}$ are isomorphic.

Proof. Apply the $\operatorname{Hom}(-, \mathbf{Z})$ functor to the short exact sequence

$$
0 \rightarrow \mathbf{T} \rightarrow \mathbf{T}_{A} \oplus \mathbf{T}_{B} \rightarrow\left(\mathbf{T}_{A} \oplus \mathbf{T}_{B}\right) / \mathbf{T} \rightarrow 0
$$

to obtain a three-term exact sequence

$$
0 \rightarrow \operatorname{Hom}\left(\mathbf{T}_{A} \oplus \mathbf{T}_{B}, \mathbf{Z}\right) \rightarrow \operatorname{Hom}(\mathbf{T}, \mathbf{Z}) \rightarrow \operatorname{Ext}^{1}\left(\left(\mathbf{T}_{A} \oplus \mathbf{T}_{B}\right) / \mathbf{T}, \mathbf{Z}\right) \rightarrow 0 .
$$

The perfect $\mathbf{T}$-equivariant bilinear pairing $\mathbf{T} \times S_{2}(\mathbf{Z}) \rightarrow \mathbf{Z}$ given by $(t, g) \mapsto$ $a_{1}(t(g))$ transforms (1) into an exact sequence

$$
0 \rightarrow S_{A} \oplus S_{B} \rightarrow S_{2}(\mathbf{Z}) \rightarrow \operatorname{Ext}^{1}\left(\left(\mathbf{T}_{A} \oplus \mathbf{T}_{B}\right) / \mathbf{T}, \mathbf{Z}\right) \rightarrow 0
$$

of $\mathbf{T}$-modules, which proves the first claim in the lemma. Finally note that if $G$ is any finite abelian group, then $\operatorname{Ext}^{1}(G, \mathbf{Z}) \approx G$ as groups, which gives the second result of the lemma.

Definition 3.4. The exponent of either of the isomorphic groups $S_{2}(\mathbf{Z}) /\left(S_{A}+\right.$ $\left.S_{B}\right)$ and $\left(\mathbf{T}_{A} \oplus \mathbf{T}_{B}\right) / \mathbf{T}$ is the congruence exponent $\tilde{r}_{A}$ of $A$ and the order of the groups is the congruence number $r_{A}$. 
Note that this definition is also symmetric with respect to $A$ and $B$, and again, the definition depends on both $A$ and $B$, unlike what the notation may suggest - we have suppressed the dependence on $B$ with the implicit understanding that $B$ has been chosen (given $A$ ). If $f$ is a newform, then by the congruence exponent/number of $A_{f}$, we mean that of $A=A_{f}^{\vee}$, with $B=I_{f} J$. In this situation, $\mathbf{T}_{A}=\mathbf{T} / I_{f}$ and $S_{A}=S_{2}(\mathbf{Z})\left[I_{f}\right]$. Recall that a subgroup $H$ of an abelian group $G$ is said to be saturated (in $G$ ) if $G / H$ is torsion-free. Now $\operatorname{Hom}\left(\mathbf{T}_{B}, \mathbf{Z}\right)$ is the unique saturated Hecke-stable complement of $S_{2}(\mathbf{Z})\left[I_{f}\right]$ in $S_{2}(\mathbf{Z})$, hence must equal $S_{2}(\mathbf{Z})\left[I_{f}\right]^{\perp}$, where we recall that $S_{2}(\mathbf{Z})\left[I_{f}\right]^{\perp}$ denotes the orthogonal complement of $S_{2}(\mathbf{Z})\left[I_{f}\right]$ in $S_{2}(\mathbf{Z})$ with respect to the Petersson inner product. Thus the congruence exponent $\tilde{r}_{A_{f}}$ is the exponent of the group

$$
\frac{S_{2}(\mathbf{Z})}{S_{2}(\mathbf{Z})\left[I_{f}\right]+S_{2}(\mathbf{Z})\left[I_{f}\right]^{\perp}},
$$

and the congruence number $r_{A_{f}}$ is its order. In particular, our definition of $r_{A_{f}}$ generalizes the definition in Section 2.1 when $A_{f}$ is an elliptic curve.

Remark 3.5. If $R$ is a subring of $\mathbf{C}$, then $S_{2}(\mathbf{Z}) \otimes_{\mathbf{Z}} R=S_{2}(R)$ (see, e.g., the discussion in [DI95, §12]). Thus the analog the group displayed in (2) with $\mathbf{Z}$ replaced by an algebraic integer ring (or even $\overline{\mathbf{Z}}$ ) gives a torsion module whose annihilator ideal meets $\mathbf{Z}$ in the ideal generated by the congruence exponent.

The following generalizes Theorem 2.1:

Theorem 3.6. Let $A$ and $B$ be as in the first paragraph of Section 3. Then:

(a) $\tilde{n}_{A} \mid \tilde{r}_{A}$.

(b) Let $\Gamma=\Gamma_{0}(N)$. If $p \nmid N$, then $\operatorname{ord}_{p}\left(\tilde{r}_{A}\right)=\operatorname{ord}_{p}\left(\tilde{n}_{A}\right)$. If $f \in S_{2}\left(\Gamma_{0}(N), \mathbf{C}\right)$ is a newform, then $\operatorname{ord}_{p}\left(\tilde{r}_{A_{f}}\right)=\operatorname{ord}_{p}\left(\tilde{n}_{A_{f}}\right)$ whenever $p^{2} \nmid N$.

We give the proof of part (a) of this theorem in Section 4 and of part (b) in Section 5. The two sections may be read independently of each other.

Remark 3.7. Let $f \in S_{2}(\Gamma, \mathbf{C})$ be a newform. When $A_{f}$ is an elliptic curve, Theorem 3.6 implies that the modular degree divides the congruence number (since for an elliptic curve, the modular degree and modular exponent are the same), and that $n_{A_{f}} \mid r_{A_{f}}^{2}$ (since for an elliptic curve, the modular number is the square of the modular exponent). In general, for a higher dimensional newform quotient, the divisibility $n_{A_{f}} \mid r_{A_{f}}^{2}$ need not hold. For example, there is a newform of degree 24 in $S_{2}\left(\Gamma_{0}(431)\right)$ such that

$$
n_{A_{f}}=\left(2^{11} \cdot 6947\right)^{2} \nmid r_{A_{f}}^{2}=\left(2^{10} \cdot 6947\right)^{2} .
$$

Note that 431 is prime and mod 2 multiplicity one fails for $J_{0}(431)$ (see [Kil02]).

\section{Proof of Theorem 3.6(a)}

Since $\operatorname{End}(J)$ preserves $A$ and $B$, we have a map $\operatorname{End}(J) \rightarrow \operatorname{End}(A) \oplus \operatorname{End}(B)$; moreover, since $A+B=J$, this map is injective. We have the following 
commutative diagram with exact rows:

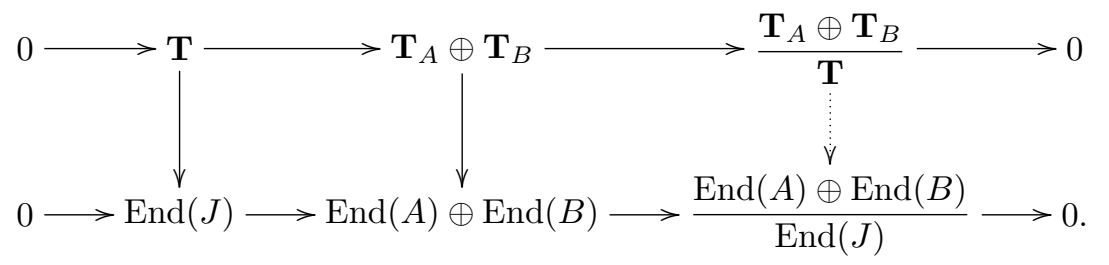

The first two vertical maps are clearly injections, and the rightmost vertical map is defined naturally so that the diagram is commutative. Let

$$
e=(1,0) \in \mathbf{T}_{A} \oplus \mathbf{T}_{B},
$$

and let $e_{1}$ and $e_{2}$ denote the images of $e$ in the groups $\left(\mathbf{T}_{A} \oplus \mathbf{T}_{B}\right) / \mathbf{T}$ and $(\operatorname{End}(A) \oplus \operatorname{End}(B)) / \operatorname{End}(J)$, respectively. Since $A \cap B$ is finite (in addition to the fact that $A+B=J)$, the two quotient groups on the right side of (3) are finite, so $e_{1}$ and $e_{2}$ have finite order.

Lemma 4.1. The element $e_{2} \in(\operatorname{End}(A) \oplus \operatorname{End}(B)) / \operatorname{End}(J)$ defined above has order $\tilde{n}_{A}$.

Proof. By the denominator of any $\varphi \in \operatorname{End}(J) \otimes \mathbf{Q}$, we mean the smallest positive integer $n$ such that $n \varphi \in \operatorname{End}(J)$. Let $\pi_{A}, \pi_{B} \in \operatorname{End}(J) \otimes \mathbf{Q}$ be projection onto $A$ and $B$, respectively. Let $n$ be the order of $e_{2}$, so $n$ is the denominator of $\pi_{A}$, which equals the denominator of $\pi_{B}$ (since $\pi_{A}+\pi_{B}=1_{J}$, so that $\left.\pi_{B}=1_{J}-\pi_{A}\right)$. We want to show that $n$ is equal to $\tilde{n}_{A}$, the exponent of $A \cap B$.

Let $i_{A}$ and $i_{B}$ be the embeddings of $A$ and $B$ into $J$, respectively. We view $n \pi_{A}$ and $n \pi_{B}$ as morphisms $J \rightarrow A$ and $J \rightarrow B$, respectively. Let $\varphi=$ $\left(n \pi_{A}, n \pi_{B}\right) \in \operatorname{Hom}(J, A \times B)$; then $\varphi \circ\left(i_{A}+i_{B}\right)=[n]_{A \times B}$. We have an exact sequence

$$
0 \rightarrow A \cap B \stackrel{x \mapsto(x,-x)}{\longrightarrow} A \times B \stackrel{i_{A}+i_{B}}{\longrightarrow} J \rightarrow 0 .
$$

Let $\Delta$ be the image of $A \cap B$. Then by exactness,

$$
[n] \Delta=\left(\varphi \circ\left(i_{A}+i_{B}\right)\right)(\Delta)=\varphi \circ\left(\left(i_{A}+i_{B}\right)(\Delta)\right)=\varphi(\{0\})=\{0\},
$$

so $n$ is a multiple of the exponent $\tilde{n}_{A}$ of $A \cap B$.

To show the opposite divisibility, consider the commutative diagram

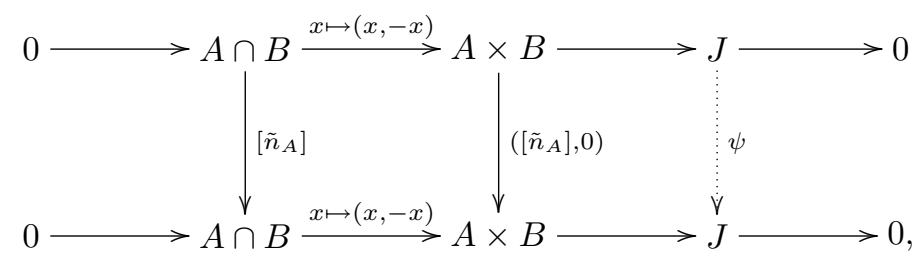


where the middle vertical map is $(a, b) \mapsto\left(\tilde{n}_{A} a, 0\right)$ and the map $\psi$ exists because $\left[\tilde{n}_{A}\right](A \cap B)=0$. But $\psi=\tilde{n}_{A} \pi_{A}$ in $\operatorname{End}(J) \otimes \mathbf{Q}$. This shows that $\tilde{n}_{A} \pi_{A} \in$ $\operatorname{End}(J)$, i.e., that $\tilde{n}_{A}$ is a multiple of the denominator $n$ of $\pi_{A}$.

Lemma 4.2. The element $e_{1} \in\left(\mathbf{T}_{A} \oplus \mathbf{T}_{B}\right) / \mathbf{T}$ has order $\tilde{r}_{A}$.

Proof. We want to show that the order $r$ of $e_{1}$ equals the exponent of $M=$ $\left(\mathbf{T}_{A} \oplus \mathbf{T}_{B}\right) / \mathbf{T}$. Since $e_{1}$ is an element of $M$, the exponent of $M$ is divisible by $r$. To obtain the reverse divisibility, consider any element $x$ of $M$. Let $(a, b) \in \mathbf{T}_{A} \oplus \mathbf{T}_{B}$ be such that its image in $M$ is $x$. By definition of $e_{1}$ and $r$, we have $(r, 0) \in \mathbf{T}$, and since $1=(1,1) \in \mathbf{T}$, we also have $(0, r) \in \mathbf{T}$. Thus $(\mathbf{T} r, 0)$ and $(0, \mathbf{T} r)$ are both subsets of $\mathbf{T}$ (i.e., in the image of $\mathbf{T}$ under the map $\left.\mathbf{T} \rightarrow \mathbf{T}_{A} \oplus \mathbf{T}_{B}\right)$, so $r(a, b)=(r a, r b)=(r a, 0)+(0, r b) \in \mathbf{T}$. This implies that the order of $x$ divides $r$. Since this is true for every $x \in M$, we conclude that the exponent of $M$ divides $r$.

Proof of Theorem 3.6(a). Since $e_{2}$ is the image of $e_{1}$ under the right-most vertical homomorphism in (3), the order of $e_{2}$ divides that of $e_{1}$. Now apply Lemmas 4.1 and 4.2 .

\section{Proof of Theorem 3.6(b)}

Let $\mathbf{T}^{\prime}$ be the saturation of $\mathbf{T}=\mathbf{Z}\left[\ldots, T_{n}, \ldots\right]$ in $\operatorname{End}(J)$, i.e.,

$$
\mathbf{T}^{\prime}=\operatorname{End}(J) \cap(\mathbf{T} \otimes \mathbf{Q}) .
$$

The quotient $\mathbf{T}^{\prime} / \mathbf{T}$ is a finitely generated abelian group because both $\mathbf{T}$ and $\operatorname{End}(J)$ are finitely generated over $\mathbf{Z}$. Since $\mathbf{T}^{\prime} / \mathbf{T}$ is also a torsion group, it is finite.

In Section 5.1, we introduce two ideals $R$ and $S$ of the Hecke algebra that are generalizations of the notions of the congruence exponent and the modular exponent respectively. We will see that $R \subset S$ and show that there is a natural injection $S / R \hookrightarrow \mathbf{T}^{\prime} / \mathbf{T}$. In Section 5.2 , we will prove that $\mathbf{T}$ and $\mathbf{T}^{\prime}$ agree locally at a maximal ideal of $\mathbf{T}$ under the condition of what we call "multiplicity one for differentials"; we also give examples where this condition does not hold. Theorem 3.6(b) itself is proved at the end of Section 5.1, by applying the results of Section 5.1 and a proposition that is proved in Section 5.2 to show that $R=S$ locally at a prime $p$ such that $p \nmid N$, or such that $p^{2} \nmid N$ for duals of newform quotients.

\subsection{The CONGRUENCE AND INTERSECTION IDEALS}

In this section, we work in slightly more generality, and take $A$ and $B$ to be as in the first paragraph of Section 3 (so $\Gamma$ can be $\Gamma_{1}(N)$, and $A$ need not be the dual of a newform quotient). Let $\pi_{A}: \mathbf{T} \rightarrow \mathbf{T}_{A}$ and $\pi_{B}: \mathbf{T} \rightarrow \mathbf{T}_{B}$ be the natural projection maps. 
Definition 5.1. With the setup as above, we define the congruence ideal as $R=\pi_{A}\left(\operatorname{ker}\left(\pi_{B}\right)\right) \subset \mathbf{T}_{A}$, and the intersection ideal as $S=\operatorname{Ann}_{\mathbf{T}_{A}}(A \cap B)$.

Lemma 5.2. We have $R \subset S$.

Proof. By definition, $R$ consists of restrictions to $A$ of Hecke operators that vanish on $B$, while $S$ consists of restrictions to $A$ of Hecke operators that vanish on $A \cap B$. The lemma follows since the image in $\mathbf{T}_{A}$ of an operator that vanishes on $B$ also vanishes on $A \cap B$.

Remark 5.3. By Lemma 5.2, we have a surjection $\mathbf{T}_{A} / R \rightarrow \mathbf{T}_{A} / S$. Note that $\pi_{A}$ induces an isomorphism

$$
\frac{\mathbf{T}}{\operatorname{ker}\left(\pi_{A}\right)+\operatorname{ker}\left(\pi_{B}\right)} \stackrel{\simeq}{\longrightarrow} \frac{\mathbf{T}_{A}}{R}
$$

and we have an isomorphism

$$
\frac{\mathbf{T}}{\operatorname{ker}\left(\pi_{A}\right)+\operatorname{ker}\left(\pi_{B}\right)} \stackrel{\simeq}{\longrightarrow} \frac{\mathbf{T}_{A} \oplus \mathbf{T}_{B}}{\mathbf{T}}
$$

obtained by sending $t \in \mathbf{T}$ to $\left(\pi_{A}(t), 0\right) \in \mathbf{T}_{A} \oplus \mathbf{T}_{B}$. Hence by Definition 3.4, the exponent of $\mathbf{T}_{A} / R$ is $\tilde{r}_{A}$ and its order is $r_{A}$. Also, $\tilde{n}_{A}$ is the exponent of $A \cap B$, and one expects that it is also the exponent of $\mathbf{T}_{A} / S$ (certainly multiplication by $\tilde{n}_{A}$ annihilates $\mathbf{T}_{A} / S$ ), which would give another proof that $\tilde{n}_{A} \mid \tilde{r}_{A}$. Instead of pursuing this, we record the following result, which will be needed later.

Proposition 5.4. If $p$ is a prime such that the localizations of $R$ and $S$ at $p$ are the same, then $\operatorname{ord}_{p}\left(\tilde{r}_{A}\right) \leq \operatorname{ord}_{p}\left(\tilde{n}_{A}\right)$.

Proof. Under the hypothesis, the surjection $\mathbf{T}_{A} / R \rightarrow \mathbf{T}_{A} / S$ is an isomorphism locally at $p$. The lemma follows from the observations above that $\tilde{r}_{A}$ is the exponent of $\mathbf{T}_{A} / R$ and that $\tilde{n}_{A}$ annihilates $\mathbf{T}_{A} / S$.

Lemma 5.5. There is a natural inclusion $S / R \hookrightarrow \mathbf{T}^{\prime} / \mathbf{T}$ of $\mathbf{T}$-modules.

Proof. We have

$\mathbf{T} \otimes \mathbf{Q} \cong\left(\mathbf{T}_{A} \otimes \mathbf{Q}\right) \oplus\left(\mathbf{T}_{B} \otimes \mathbf{Q}\right) \subset(\operatorname{End}(A) \otimes \mathbf{Q}) \oplus(\operatorname{End}(B) \otimes \mathbf{Q}) \cong \operatorname{End}(J) \otimes \mathbf{Q}$,

which we use to view $\mathbf{T}$ and $\mathbf{T}_{A}$ as sitting inside $\operatorname{End}(J) \otimes \mathbf{Q}$. Also, the groups $\operatorname{End}(J)$ and $\mathbf{T}^{\prime}$ sit naturally in $\operatorname{End}(J) \otimes \mathbf{Q}$. By definition, $R=\mathbf{T}_{A} \cap \mathbf{T}$. Since an endomorphism of $A \times B$ factors through $A \times B \rightarrow J$ if and only if it kills $A \cap B$ embedded in $A \times B$ via $x \mapsto(x,-x)$, we have that $S=\mathbf{T}_{A} \cap \operatorname{End}(J)$ and this equals $\mathbf{T}_{A} \cap \mathbf{T}^{\prime}$ (since a suitable multiple of any element of $\mathbf{T}_{A}$ lands in $\mathbf{T}$, when both are viewed as subgroups of $\mathbf{T} \otimes \mathbf{Q} \subset \operatorname{End}(J) \otimes \mathbf{Q})$. Hence we have $R=S \cap \mathbf{T}$ with intersection taken inside $\mathbf{T}^{\prime} \subset \operatorname{End}(J) \otimes \mathbf{Q}$. Thus

$$
S / R=S /(S \cap \mathbf{T}) \cong(S+\mathbf{T}) / \mathbf{T} \hookrightarrow \mathbf{T}^{\prime} / \mathbf{T} .
$$


If $\mathfrak{m}$ is a maximal ideal of $\mathbf{T}$, then we say that two Hecke modules, with one contained in the other, agree locally at $\mathfrak{m}$ if their localizations at $\mathfrak{m}$ are the same. Let $I_{A}$ denote the kernel of the map $\mathbf{T} \rightarrow \mathbf{T}_{A}$. As an immediate consequence of Lemma 5.5, we have:

Proposition 5.6. If $\mathfrak{m}$ is a maximal ideal of $\mathbf{T}$ containing $I_{A}$ that is not in $\operatorname{Supp}_{\mathbf{T}}\left(\mathbf{T}^{\prime} / \mathbf{T}\right)$, then the corresponding maximal ideal $\mathfrak{m} / I_{A}$ of $\mathbf{T}_{A}$ is not in the support of $S / R$, i.e., if $\mathbf{T}$ and $\mathbf{T}^{\prime}$ agree locally at $\mathfrak{m}$, then $R$ and $S$ also agree locally at $\mathfrak{m} / I_{A}$.

REMARK 5.7. The ring

$$
\mathbf{T}^{\prime \prime}=\operatorname{End}(J) \cap\left(\mathbf{T}_{A} \times \mathbf{T}_{B}\right)=\mathbf{T}^{\prime} \cap\left(\mathbf{T}_{A} \times \mathbf{T}_{B}\right)
$$

is often of interest, where the intersection is taken in $\operatorname{End}(J) \otimes \mathbf{Q}$. We proved above that there is a natural inclusion $S / R \hookrightarrow \mathbf{T}^{\prime} / \mathbf{T}$. This inclusion yields an isomorphism $S / R \stackrel{\sim}{\longrightarrow} \mathbf{T}^{\prime \prime} / \mathbf{T}$, as is clear from the "if and only if" statement in the proof of Lemma 5.5. The ideals $R$ and $S$ are equal if the rings $\mathbf{T}$ and $\mathbf{T}^{\prime \prime}$ coincide. Even when $\mathbf{T}^{\prime}$ is bigger than $\mathbf{T}$, its subring $\mathbf{T}^{\prime \prime}$ may be not far from $\mathbf{T}$.

The following lemma and proposition will not be used in the proof of Theorem 3.6(b), but they are of interest from the point of view of multiplicity one.

Lemma 5.8. Let $p$ be a prime and let $\mathfrak{m}$ be a maximal ideal of $\mathbf{T}$ with residue characteristic $p$. Suppose $\mathfrak{m}$ satisfies the multiplicity one condition (i.e., $J[\mathfrak{m}]$ is of dimension two over $\mathbf{T} / \mathfrak{m})$. Then the completions of $\mathbf{T}$ and $\mathbf{T}^{\prime}$ at $\mathfrak{m}$ are isomorphic.

Proof. As in [Maz77, p.92], consider the Tate module $\operatorname{Ta}_{\mathfrak{m}}(J)$, which is the Pontryagin dual of the $\mathfrak{m}$-divisible group associated to $J(\overline{\mathbf{Q}})$. Since $J[\mathfrak{m}]$ is of dimension two over $\mathbf{T} / \mathfrak{m}$, it follows that $\operatorname{Ta}_{\mathfrak{m}}(J)$ is free of rank 2 over $\mathbf{T}_{\mathfrak{m}}$, where the subscript denotes completion (see, e.g., [Til97, p. 332-333]). If $r$ is an element of $\mathbf{T}_{\mathfrak{m}}^{\prime}$, then $r$ operates $\mathbf{T}_{\mathfrak{m}}$-linearly on $\operatorname{Ta}_{\mathfrak{m}}(J)$, and thus may be viewed as a $2 \times 2$ matrix with entries in $\mathbf{T}_{\mathfrak{m}}$. Further, some non-zero integer multiple of $r$ operates on $\operatorname{Ta}_{\mathfrak{m}}(J)$ as an element of $\mathbf{T}_{\mathfrak{m}}$, i.e., as a scalar. Thus $r$ must be a scalar to start with, i.e., actually lies in $\mathbf{T}_{\mathfrak{m}}$. Hence $\mathbf{T}_{\mathfrak{m}}^{\prime}=\mathbf{T}_{\mathfrak{m}}$ as claimed.

Proposition 5.9. Let $p$ be a prime such that all maximal ideals $\mathfrak{m}$ of $\mathbf{T}$ with residue characteristic $p$ that contain $I_{A}$ satisfy multiplicity one. Then $\operatorname{ord}_{p}\left(\tilde{r}_{A}\right)=\operatorname{ord}_{p}\left(\tilde{n}_{A}\right)$.

Proof. This follows from Lemma 5.8, Lemma 5.5, Proposition 5.4, and Theorem 3.6(a).

Proposition 5.10. Let $\Gamma=\Gamma_{0}(N)$. Let $p$ be a prime such that $p^{2} \nmid N$, and let $\mathfrak{m}$ be a maximal ideal of $\mathbf{T}$ with residue characteristic $p$. If $p \mid N$, then assume that $I_{f} \subseteq \mathfrak{m}$ for some newform $f$. Then $\mathbf{T}$ and $\mathbf{T}^{\prime}$ agree locally at $\mathfrak{m}$. 
Since the proof of this proposition is rather technical, we have postponed it to Section 5.2. Admitting this proposition, we may now finish the proof of Theorem 3.6(b).

Proof of Theorem 3.6(b). Recall that $A$ and $B$ are abelian subvarieties of $J=$ $J_{0}(N)$ such that $A+B=J, A \cap B$ is finite, and every endomorphism of $J$ over $\mathbf{Q}$ preserves $A$ and $B$.

We first want to show that if a prime $p$ does not divide $N$, then $\operatorname{ord}_{p}\left(\tilde{r}_{A}\right)=$ $\operatorname{ord}_{p}\left(\tilde{n}_{A}\right)$. In view of Theorem 3.6(a) and Proposition 5.4, it suffices to check that $R$ and $S$ coincide locally at $p$. By Proposition 5.6, it suffices to check that $\mathbf{T}$ and $\mathbf{T}^{\prime}$ are locally equal at all maximal ideals that divide $p$. If $p \nmid N$, then this follows from Proposition 5.10, which proves part of Theorem 3.6(b).

It remains to show that if $f \in S_{2}\left(\Gamma_{0}(N), \mathbf{C}\right)$ is a newform and $p \| N$, then $\operatorname{ord}_{p}\left(\tilde{r}_{A_{f}}\right)=\operatorname{ord}_{p}\left(\tilde{n}_{A_{f}}\right)$. Note that the Hecke algebra $\mathbf{T}$ acts on $S / R$ through its quotient $\mathbf{T}_{A_{f}^{\vee}}=\mathbf{T} / \mathrm{Ann}_{\mathbf{T}} A_{f}^{\vee}$ since the action of $\mathbf{T}$ on $R$ and on $S$ factors through this quotient. Thus, in view of Theorem 3.6(a) and Proposition 5.4, it suffices to check that $R$ and $S$ coincide locally at maximal ideals of $\mathbf{T}$ that divide $p$ and contain $\mathrm{Ann}_{\mathbf{T}} A_{f}^{\vee}=I_{f}$ (the equality follows since $I_{f}$ is saturated). But this follows from Proposition 5.6 and Proposition 5.10.

\subsection{Multiplicity One FOR Differentials}

This section is devoted to the proof of Proposition 5.10 as well as to discuss the notion of multiplicity one for differentials (Definition 5.13). In this section, we take $\Gamma=\Gamma_{0}(N)$.

Let $p$ be a prime such that $p^{2} \nmid N$. Let $M_{0}(N)$ denote the compactified coarse moduli scheme associated to $\Gamma_{0}(N)$ (as in [DR73, § IV.3]) over $\mathbf{Z}_{p}$, and let $X_{0}(N) \mathbf{z}_{p}$ denote its minimal regular resolution obtained by suitable blow-up of the points $j=0,1728$ in characteristic dividing $N$, when they are supersingular (cf. [Maz77, p.63]). Let $\Omega_{X_{0}(N) / \mathbf{Z}_{p}}$ denote the relative dualizing sheaf of $X_{0}(N) \mathbf{z}_{p}$ over $\mathbf{Z}_{p}$ (it is the sheaf of regular differentials as in [MR91, $\S 7])$. We denote by $X_{0}(N)_{\mathbf{F}_{p}}$ the special fiber of $X_{0}(N) \mathbf{z}_{p}$ at the prime $p$ and by $\Omega_{X_{0}(N) / \mathbf{F}_{p}}$ the relative dualizing sheaf of $X_{0}(N) \mathbf{F}_{p}$ over $\mathbf{F}_{p}$.

The usual Hecke operators and the Atkin-Lehner involutions (corresponding to primes dividing $N$ ) of $J_{0}(N)$ over $\mathbf{Q}$ extend uniquely to act on the base change to $\mathbf{Z}_{p}$ of the Néron model of $J_{0}(N)$, which we denote $J_{\mathbf{Z}_{p}}$. The natural morphism $\operatorname{Pic}_{X_{0}(N) / \mathbf{z}_{p}}^{0} \rightarrow J_{\mathbf{Z}_{p}}$ identifies $\operatorname{Pic}_{X_{0}(N) / \mathbf{z}_{p}}^{0}$ with the identity component of $J_{\mathbf{Z}_{p}}$ (see, e.g., [BLR90, §9.4-9.5]). Passing to tangent spaces along the identity section over $\mathbf{Z}_{p}$, we obtain an isomorphism $H^{1}\left(X_{0}(N) \mathbf{z}_{p}, \mathcal{O}_{X_{0}(N) \mathbf{z}_{p}}\right) \cong \operatorname{Tan}\left(J_{\mathbf{z}_{p}}\right)$. Using Grothendieck duality, one gets an isomorphism $\operatorname{Cot}\left(J_{\mathbf{z}_{p}} \cong H^{0}\left(X_{0}(N) \mathbf{z}_{p}, \Omega_{X_{0}(N) / \mathbf{z}_{p}}\right)\right.$, where $\operatorname{Cot}\left(J_{\mathbf{z}_{p}}\right)$ is the cotangent space at the identity section (cf. [Maz78, p. 140]). Now the Hecke operators and the Atkin-Lehner involutions act on $\operatorname{Cot}\left(J_{\mathbf{Z}_{p}}\right)$, and hence via the last isomorphism above, we get an action of the Hecke operators and the Atkin-Lehner involutions on $H^{0}\left(X_{0}(N) \mathbf{z}_{p}, \Omega_{X_{0}(N) / \mathbf{z}_{p}}\right)$. Following the proof of 
Prop. 3.3 on p. 68 of [Maz77], specialization induces an isomorphism

$$
\mathrm{H}^{0}\left(X_{0}(N)_{\mathbf{F}_{p}}, \Omega_{X_{0}(N) / \mathbf{F}_{p}}\right) \cong \mathrm{H}^{0}\left(X_{0}(N) \mathbf{z}_{p}, \Omega_{X_{0}(N) / \mathbf{z}_{p}}\right) \otimes \mathbf{z}_{p} \mathbf{F}_{p} .
$$

In this way, we get an action of the Hecke operators and the Atkin-Lehner involutions on $H^{0}\left(X_{0}(N)_{\mathbf{F}_{p}}, \Omega_{X_{0}(N) / \mathbf{F}_{p}}\right)$ as well.

The following lemma is implicit in [Maz77, p. 95].

Lemma 5.11 (Mazur). Let $\mathfrak{m}$ be a maximal ideal of $\mathbf{T}$ of residue characteristic $p$ (recall that $\left.p^{2} \nmid N\right)$. Suppose

$$
\operatorname{dim}_{\mathbf{T} / \mathfrak{m}} \mathrm{H}^{0}\left(X_{0}(N)_{\mathbf{F}_{p}}, \Omega_{X_{0}(N) / \mathbf{F}_{p}}\right)[\mathfrak{m}] \leq 1 .
$$

Then $\mathbf{T}$ and $\mathbf{T}^{\prime}$ agree locally at $\mathfrak{m}$.

Proof. Let $M$ denote the group $H^{1}\left(X_{0}(N) \mathbf{z}_{p}, \mathcal{O}_{X_{0}(N)}\right)$, where $\mathcal{O}_{X_{0}(N)}$ is the structure sheaf of $X_{0}(N)$. As explained in [Maz77, p. 95], we have an action of $\operatorname{End}_{\mathbf{Q}} J_{0}(N)$ on $M$, and the action of $\mathbf{T}$ on $M$ via the inclusion $\mathbf{T} \subset \operatorname{End}_{\mathbf{Q}} J_{0}(N)$ is faithful, so likewise for the action by $\mathbf{T}^{\prime}$. Hence we have an injection $\phi: \mathbf{T}^{\prime} \hookrightarrow \operatorname{End}_{\mathbf{T}} M$. Suppose $\mathfrak{m}$ is a maximal ideal of $\mathbf{T}$ that satisfies the hypotheses of the lemma. To prove that $\mathbf{T}_{\mathfrak{m}}=\mathbf{T}_{\mathfrak{m}}^{\prime}$ it suffices to prove the following claim:

Claim: The map $\left.\phi\right|_{\mathbf{T}}$ is surjective locally at $\mathfrak{m}$.

Proof. It suffices to show that $M$ is generated by a single element over $\mathbf{T}$ locally at $\mathfrak{m}$, and in turn, by Nakayama's lemma, it suffices to check that the dimension of the $\mathbf{T} / \mathfrak{m}$-vector space $M / \mathfrak{m} M$ is at most one. Now $M / \mathfrak{m} M$ is dual to $H^{0}\left(X_{0}(N)_{\mathbf{F}_{p}}, \Omega_{X_{0}(N) / \mathbf{F}_{p}}\right)[\mathfrak{m}]$. Since we are assuming that $\operatorname{dim}_{\mathbf{T} / \mathfrak{m}} H^{0}\left(X_{0}(N)_{\mathbf{F}_{p}}, \Omega_{X_{0}(N) / \mathbf{F}_{p}}\right)[\mathfrak{m}] \leq 1$, we have $\operatorname{dim}_{\mathbf{T} / \mathfrak{m}}(M / \mathfrak{m} M) \leq 1$, which proves the claim.

REMARK 5.12. Note that Lemma 5.8 may provide an alternate route to the conclusion of the previous lemma (sometimes one can prove multiplicity one for a maximal ideal without relying on multiplicity one for differentials, e.g., see [Dia97]). Observe that in the proofs of Lemmas 5.11 and 5.8, all we needed was (locally) a non-zero free $\mathbf{T}$-module (of finite rank, say) that is attached functorially to $J$. In Lemma 5.11, the module we used was $H^{1}\left(X_{0}(N) \mathbf{z}_{p}, \mathcal{O}_{X_{0}(N)}\right)$; locally, it is free because its reduction modulo $\mathfrak{m}$ is of the same dimension as its generic rank (namely 1 ). In Lemma 5.8, we used the $\mathfrak{m}$-adic Tate module, whose reduction $\bmod \mathfrak{m}$ is of the same dimension as its generic rank (namely 2).

Definition 5.13. If $\mathfrak{m}$ is a maximal ideal of the Hecke algebra $\mathbf{T}$ of residue characteristic $p$, we say that $\mathfrak{m}$ satisfies multiplicity one for differentials if

$$
\operatorname{dim}_{\mathbf{T} / \mathfrak{m}}\left(\mathrm{H}^{0}\left(X_{0}(N)_{\mathbf{F}_{p}}, \Omega_{X_{0}(N) / \mathbf{F}_{p}}\right)[\mathfrak{m}]\right)=1 .
$$


The above condition, which first appeared in [Maz77], plays an important role in several places, including Wiles's proof of Fermat's last theorem (see [Wil95, Lemma 2.2]). It is has been used to prove multiplicity one for $\mathfrak{m}$ (as in Section 2.2) and Gorensteinness of the completion of $\mathbf{T}$ at $\mathfrak{m}$ (under certain hypotheses; see, e.g., [Til97]).

\subsubsection{FAilure OF MULTiPlicity ONE FOR DifFERENTIALS}

In this section, we digress to discuss examples of failure of multiplicity one for differentials. The reader interested in the proof of Proposition 5.10 may jump to Section 5.2.2 below.

By Lemma 5.11, if $p^{2} \nmid N$ and if the multiplicity one condition for differentials holds at $\mathfrak{m}$, then $\mathbf{T}$ and $\mathbf{T}^{\prime}$ agree locally at $\mathfrak{m}$. It is thus of interest to compute the quotient group $\mathbf{T}^{\prime} / \mathbf{T}$ for various $N$. We compute this index in Sage $\left[\mathrm{S}^{+} 09\right]$. and obtain Table 2, where the first column contains $N$ for $N \leq 325$ and the second column contains the quotient group $\mathbf{T}^{\prime} / \mathbf{T}$, where $C_{n}$ denotes a cyclic group of order $n$.

In each case in which a prime $p$ divides $\left[\mathbf{T}^{\prime}: \mathbf{T}\right]$ but $p^{2} \nmid N$, Lemma 5.11 implies that there is some maximal ideal $\mathfrak{m}$ of $\mathbf{T}$ of residue characteristic $p$ for which multiplicity one for differentials does not hold. For example, when $N=46$, we find that $\left[\mathbf{T}^{\prime}: \mathbf{T}\right]=2$, and $2^{2} \nmid N$; thus there is a maximal ideal $\mathfrak{m}$ of $\mathbf{T}$ of residue characteristic 2 for which multiplicity one for differentials does not hold.

In Table 2, we observe that whenever $p$ divides $\left[\mathbf{T}^{\prime}: \mathbf{T}\right]$, then $p=2$ or $p^{2} \mid N$. This raises the question: is it true that if $p$ is odd and $p^{2} \nmid N$, then multiplicity one for differentials holds for maximal ideals $\mathfrak{m}$ of $\mathbf{T}$ of residue characteristic $p$ ? Lemma 5.20 below gives an affirmative answer in one direction (the other direction is usually easy), but under the hypothesis that if $p \mid N$ then $U_{p}$ acts as a non-zero scalar on $H^{0}\left(X_{0}(N)_{\mathbf{F}_{p}}, \Omega_{X_{0}(N) / \mathbf{F}_{p}}\right)[\mathfrak{m}]$.

\subsubsection{Proof of Proposition 5.10}

The main point is to prove that the hypothesis

$$
\operatorname{dim}_{\mathbf{T} / \mathfrak{m}} \mathrm{H}^{0}\left(X_{0}(N)_{\mathbf{F}_{p}}, \Omega_{X_{0}(N) / \mathbf{F}_{p}}\right)[\mathfrak{m}] \leq 1
$$

of Lemma 5.11 holds for suitable maximal ideals $\mathfrak{m}$. This is achieved in Lemma 5.20 below, whose proof requires an Eichler-Shimura type relation for $U_{p}$ (Lemma 5.15 below). We obtain this relation by modifying the argument in [Wil80, $\S 5]$, which is in the $\Gamma_{1}(N)$ context, to the $\Gamma_{0}(N)$ situation. Let $L$ denote the maximal unramified extension of $\mathbf{Q}_{p}$ and let $\mathcal{O}_{L}$ denote the ring of integers of $L$. For the sake of completeness, we state below a lemma that is well known (e.g., it is used implicitly in [Wil80, p. 18]); the proof was indicated to us by F. Calegari. 
Table 2: Nonzero Quotients $\mathbf{T}^{\prime} / \mathbf{T}$ for $N \leq 325$

\begin{tabular}{|c|c|c|c|c|c|}
\hline 44 & $C_{2}$ & 160 & $C_{2}^{3} \oplus C_{4} \oplus C_{8}$ & 245 & $C_{7}^{2}$ \\
\hline 46 & $C_{2}$ & 162 & $C_{3}^{4}$ & 248 & $C_{2}^{7} \oplus C_{4} \oplus C_{8}$ \\
\hline 54 & $C_{3}$ & 164 & $C_{2}^{3}$ & 250 & $C_{5}^{8}$ \\
\hline 56 & $C_{2}$ & 166 & $C_{2}$ & 252 & $C_{2}^{2} \oplus C_{6}^{3} \oplus C_{12}$ \\
\hline 60 & $C_{2}$ & 168 & $C_{2}^{5} \oplus C_{4}$ & 254 & $C_{2}^{2}$ \\
\hline 62 & $C_{2}$ & 169 & $C_{13}$ & 256 & $C_{2}^{3} \oplus C_{4}^{2} \oplus C_{8}^{2} \oplus C_{16}$ \\
\hline 64 & $C_{2}$ & 171 & $C_{3}^{2}$ & 260 & $C_{2}^{6}$ \\
\hline 68 & $C_{2}$ & 172 & $C_{2}^{3}$ & 261 & $C_{3}^{4}$ \\
\hline 72 & $C_{2}$ & 174 & $C_{2}$ & 262 & $C_{2}^{2}$ \\
\hline 76 & $C_{2}$ & 175 & $C_{5}$ & 264 & $C_{2}^{7} \oplus C_{4}^{3}$ \\
\hline 78 & $C_{2}$ & 176 & $C_{2}^{2} \oplus C_{4}^{2} \oplus C_{8}$ & 268 & $C_{2}^{5}$ \\
\hline 80 & $C_{4}$ & 180 & $C_{2} \oplus C_{6}^{2}$ & 270 & $C_{3}^{9} \oplus C_{6}^{2}$ \\
\hline 84 & $C_{2}$ & 184 & $C_{2}^{5} \oplus C_{4} \oplus C_{8}$ & 272 & $C_{2}^{3} \oplus C_{4}^{4} \oplus C_{8}$ \\
\hline 88 & $C_{2} \oplus C_{4}$ & 186 & $C_{2}^{2}$ & 275 & $C_{5}^{4}$ \\
\hline 92 & $C_{2}^{2} \oplus C_{4}$ & 188 & $C_{2}^{4} \oplus C_{4}^{2}$ & 276 & $C_{2}^{7} \oplus C_{4}^{2}$ \\
\hline 94 & $C_{2}^{2}$ & 189 & $C_{3}^{5}$ & 278 & $C_{2}$ \\
\hline 96 & $C_{2}^{3}$ & 190 & $C_{2}^{3}$ & 279 & $C_{3}^{4}$ \\
\hline 99 & $C_{3}^{2}$ & 192 & $C_{2}^{3} \oplus C_{4}^{3} \oplus C_{8}$ & 280 & $C_{2}^{7} \oplus C_{4}^{3}$ \\
\hline 104 & $C_{2}^{2}$ & 196 & $C_{14}$ & 282 & $C_{2}^{2}$ \\
\hline 108 & $C_{3}^{2} \oplus C_{6}$ & 198 & $C_{3}^{4}$ & 284 & $C_{2}^{6} \oplus C_{4}^{3}$ \\
\hline 110 & $C_{2}$ & 200 & $C_{2}^{3} \oplus C_{10}$ & 286 & $C_{2}^{4}$ \\
\hline 112 & $C_{2} \oplus C_{4}$ & 204 & $C_{2}^{5}$ & 288 & $C_{2}^{7} \oplus C_{4}^{3} \oplus C_{12} \oplus C_{24}$ \\
\hline 116 & $C_{2}^{2}$ & 206 & $C_{2}^{2}$ & 289 & $C_{17}^{2}$ \\
\hline 118 & $C_{2}$ & 207 & $C_{3}^{4}$ & 290 & $C_{2}$ \\
\hline 120 & $C_{2}^{3} \oplus C_{4}$ & 208 & $C_{2}^{2} \oplus C_{4}^{3}$ & 292 & $C_{2}^{5}$ \\
\hline 124 & $C_{2}^{2} \oplus C_{4}$ & 210 & $C_{2}$ & 294 & $C_{7}^{4}$ \\
\hline 125 & $C_{5}^{2}$ & 212 & $C_{2}^{4}$ & 296 & $C_{2}^{6} \oplus C_{4}^{2}$ \\
\hline 126 & $C_{3} \oplus C_{6}$ & 214 & $C_{2}$ & 297 & $C_{3}^{8} \oplus C_{9}$ \\
\hline 128 & $C_{2} \oplus C_{4} \oplus C_{8}$ & 216 & $C_{3} \oplus C_{6}^{5} \oplus C_{12}$ & 300 & $C_{2}^{2} \oplus C_{10}^{3}$ \\
\hline 132 & $C_{2}^{3}$ & 220 & $C_{2}^{5} \oplus C_{4}$ & 302 & $C_{2}^{3}$ \\
\hline 135 & $C_{3}^{3}$ & 224 & $C_{2}^{5} \oplus C_{4}^{2} \oplus C_{8}$ & 304 & $C_{2}^{4} \oplus C_{4}^{4} \oplus C_{8}$ \\
\hline 136 & $C_{2}^{2} \oplus C_{4}$ & 225 & $C_{5}$ & 306 & $C_{3}^{6}$ \\
\hline 140 & $C_{2}^{3}$ & 228 & $C_{2}^{5}$ & 308 & $C_{2}^{7}$ \\
\hline 142 & $C_{2}^{3}$ & 230 & $C_{2}^{2}$ & 310 & $C_{2}^{3}$ \\
\hline 144 & $C_{2}^{3} \oplus C_{4}$ & 232 & $C_{2}^{4} \oplus C_{4}^{2}$ & 312 & $C_{2}^{11} \oplus C_{4}^{2} \oplus C_{8}$ \\
\hline 147 & $C_{7}$ & 234 & $C_{3}^{2} \oplus C_{6}^{2}$ & 315 & $C_{3}^{6}$ \\
\hline 148 & $C_{2}^{2}$ & 236 & $C_{2}^{5} \oplus C_{4}$ & 316 & $C_{2}^{6} \oplus C_{4}^{2}$ \\
\hline 150 & $C_{5}$ & 238 & $C_{2}^{4}$ & 318 & $C_{2}^{4}$ \\
\hline 152 & $C_{2}^{3} \oplus C_{4}$ & 240 & $C_{2}^{7} \oplus C_{4}^{3} \oplus C_{8}$ & 320 & $C_{2}^{6} \oplus C_{4}^{3} \oplus C_{8}^{3} \oplus C_{16}$ \\
\hline 153 & $C_{3}$ & 242 & $C_{11}^{2}$ & 322 & $C_{2}^{2}$ \\
\hline 156 & $C_{2}^{3} \oplus C_{4}$ & 243 & $C_{3}^{4} \oplus C_{9}^{2}$ & 324 & $C_{3}^{7} \oplus C_{6}^{3} \oplus C_{18}$ \\
\hline 158 & $C_{2}^{2}$ & 244 & $C_{2}^{4}$ & 325 & $C_{5}^{3}$ \\
\hline
\end{tabular}


LEMma 5.14. Let $E$ be an elliptic curve over $\mathcal{O}_{L}$ with good ordinary reduction. Then the subgroup schemes of $E$ of order $p$ are $p$ copies of $\mathbf{Z} / p \mathbf{Z}$ and one copy of $\boldsymbol{\mu}_{p}$.

Proof. Let $G=E[p]$, and consider its connected-étale sequence

$$
0 \rightarrow G^{0} \rightarrow G \rightarrow G^{\mathrm{et}} \rightarrow 0 .
$$

Now $G^{0}$ is in the kernel of the reduction map, and we know that the reduction of $E[p]$ has non-trivial order. Hence $G^{\text {et }}$ is non-trivial. By Cartier duality, $G^{0}$ is also non-trivial. Hence $G^{\text {et }}$ is a $\mathbf{Z} / p \mathbf{Z}$ and by duality, $G^{0}$ is a $\boldsymbol{\mu}_{p}$. Thus one of the subgroup schemes of $E$ of order $p$ is a copy of $\boldsymbol{\mu}_{p}$. Let $H$ be any other subgroup scheme of $E$ of order $p$. Then $H^{0}$ has to be trivial, since otherwise $H=H^{0}$ is a nontrivial subgroup scheme of $G^{0}=\boldsymbol{\mu}_{p}$, hence is equal to $G^{0}=\boldsymbol{\mu}_{p}$, which has already been accounted for. Thus $H$ is étale, and hence is a copy of $\mathbf{Z} / p \mathbf{Z}$. The lemma follows, since there are $p+1$ subgroup schemes of order $p$ in $E[p]$, hence in $E$.

We assume that $p \| N$ until just after the proof of Lemma 5.18. Let $M=N / p$. We will use the superscript $h$ to denote the subscheme of $M_{0}(N)$ obtained by removing the supersingular points in characteristic $p$. Follow-

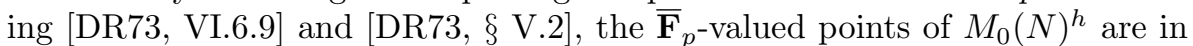
one-to-one correspondence with isomorphism classes of triples consisting of

(a) a generalized elliptic curve $E$ over $\overline{\mathbf{F}}_{p}$, whose smooth locus we denote $E^{\mathrm{sm}}$, (b) a subgroup of $E^{\mathrm{sm}}[p]$ isomorphic to $\boldsymbol{\mu}_{p}$ or to $\mathbf{Z} / p \mathbf{Z}$, and

(c) a subgroup $\mathbf{Z} / M \mathbf{Z}$ of $E^{\mathrm{sm}}[M]$,

such that the subgroup generated by the subgroups in $(b)$ and $(c)$ above meets every irreducible component of every geometric fiber of $E$ over $\overline{\mathbf{F}}_{p}$. Also, $M_{0}(N)_{\overline{\mathbf{F}}_{p}}$ has two irreducible components, which may be described according as whether the subgroup in (b) is isomorphic to $\boldsymbol{\mu}_{p}$ or to $\mathbf{Z} / p \mathbf{Z}$. As mentioned earlier, $X_{0}(N)_{\overline{\mathbf{F}}_{p}}$ is obtained from $M_{0}(N)_{\overline{\mathbf{F}}_{p}}$ by suitable blowups and consists of two copies of $X_{0}(M)_{\overline{\mathbf{F}}_{p}}$ identified at supersingular points, along with some copies of $\mathbf{P}^{1}$ (see the description of $X_{0}(N)_{\overline{\mathbf{F}}_{p}}$ on p. 175-177 of [Maz77] for details). One of the copies of $X_{0}(M)_{\overline{\mathbf{F}}_{p}}$ corresponds to the irreducible component of $M_{0}(N)_{\overline{\mathbf{F}}_{p}}$ where the subgroup in (b) is isomorphic to $\mathbf{Z} / p \mathbf{Z}$; we denote this copy by $C_{0}$. The other copy of $X_{0}(M)_{\overline{\mathbf{F}}_{p}}$ corresponds to the irreducible component of $M_{0}(N)_{\overline{\mathbf{F}}_{p}}$ where the subgroup in (b) is isomorphic to $\boldsymbol{\mu}_{p}$, and contains the cusp $\infty$; we denote this copy by $C_{1}$. We denote the copies (if any) of $\mathbf{P}^{1}$ by $C_{2}, \ldots, C_{r}$, where $r$ is one less than the total number of irreducible components of $X_{0}(N)_{\overline{\mathbf{F}}_{p}}$.

The usual endomorphisms $U_{p}$ and $W_{p}$ of $J_{0}(N)$ over $\mathbf{Q}$ can be extended by base change to $L$, and extend uniquely to act on the Néron model of $J_{0}(N)$ over $\mathcal{O}_{L}$. Since the formation of Néron models is compatible with completions and unramified base change, this action is compatible with the already defined 
action on the the Néron model of $J_{0}(N)$ over $\mathbf{Z}_{p}$. The identity component of the special fiber of the Néron model of $J_{0}(N)$ over $\mathcal{O}_{L}$ is $\operatorname{Pic}_{X_{0}(N) / \overline{\mathbf{F}}_{p}}^{0}$, whose maximal abelian variety quotient is $\prod_{i=0}^{r} \operatorname{Pic}_{C_{i} / \overline{\mathbf{F}}_{p}}^{0}$ (cf. [DR73, I.3.7] and [BLR90, $\S 9.2$, Example 8]). Thus we get an action of $U_{p}$ and $W_{p}$ on $\operatorname{Pic}_{X_{0}(N) / \overline{\mathbf{F}}_{p}}^{0}$ and on $\prod_{i=0}^{r} \operatorname{Pic}_{C_{i} / \overline{\mathbf{F}}_{p}}^{0}$. Let Frob ${ }_{p}$ denote the Frobenius morphism on $C_{0 / \overline{\mathbf{F}}_{p}}$.

LEMmA 5.15. The endomorphisms $U_{p}$ and $W_{p}$ of $\prod_{i=0}^{r} \operatorname{Pic}_{C_{i} / \overline{\mathbf{F}}_{p}}^{0}$ satisfy $U_{p}=$ Frob $_{p}+(p-1) W_{p}$ on $\operatorname{Pic}_{C_{0} / \overline{\mathbf{F}}_{p}}^{0}$.

Proof. The proof is a modification of the proof of Theorem 5.3 in [Wil80], along with some details borrowed from the proof of Theorem 5.16 in B. Conrad's appendix to [RS01].

It suffices to check the desired identity on a Zariski dense subset of $\operatorname{Pic}^{0}{ }_{C_{0}} / \overline{\mathbf{F}}_{p}\left(\overline{\mathbf{F}}_{p}\right)=J\left(C_{0}\right)\left(\overline{\mathbf{F}}_{p}\right)$, where $J\left(C_{0}\right)$ is the Jacobian of $C_{0}$. If $g$ is the genus of $C_{0}$, then fixing a base point, we get a surjection $C_{0}^{g} \rightarrow J\left(C_{0}\right)$. Hence if $U$ is any dense open subset of $C_{0}\left(\overline{\mathbf{F}}_{p}\right)$, then $U^{g}$ hits a Zariski dense subset of $J\left(C_{0}\right)\left(\overline{\mathbf{F}}_{p}\right)$. Taking $U$ to be the ordinary locus of $C_{0}\left(\overline{\mathbf{F}}_{p}\right)$, it thus suffices to prove the desired identity on divisors of the form $(Q)-\left(Q^{\prime}\right)$, where the elliptic curves corresponding to $Q, Q^{\prime} \in C_{0}\left(\overline{\mathbf{F}}_{p}\right)$ are ordinary.

Let $\mathcal{M}_{0}(N)$ denote the algebraic stack over $\mathcal{O}_{L}$ associated to $\Gamma_{0}(N)$ by [DR73, IV.3.3, IV.4.2], whose associated coarse moduli scheme is $M_{0}(N)$ (over $\left.\mathcal{O}_{L}\right)$. Let $\pi: \mathcal{M}_{0}(N) \rightarrow M_{0}(N)$ denote the associated natural map. If $k=\overline{\mathbf{F}}_{p}$ or an algebraic closure of $L$, then $\pi$ is an isomorphism on $k$ valued points, and so we will often identify points on $M_{0}(N)(k)$ with points on $\mathcal{M}_{0}(N)(k)$. Let $Q$ be an ordinary point on $C_{0}\left(\overline{\mathbf{F}}_{p}\right)$. Then $Q$ is given by a triple $(\bar{E}, \bar{C}, \bar{D})$, where $\bar{E}$ is an ordinary elliptic curve over $\overline{\mathbf{F}}_{p}, \bar{C}$ is a subgroup isomorphic to $\mathbf{Z} / p \mathbf{Z}$, and $\bar{D}$ is a subgroup isomorphic to $\mathbf{Z} / M \mathbf{Z}$. We can choose a Weierstrass model $E \hookrightarrow \mathbf{P}_{\mathcal{O}_{L}}^{2}$ lifting $\bar{E}$; then $E$ is canonically an elliptic curve by [KM85, Chap. 2]. By Lemma 5.14 and its proof, there is a subgroup $C$ of $E$ isomorphic to $\mathbf{Z} / p \mathbf{Z}$ that lifts $\bar{C}$. Also, as argued in [RS01, p. 219], there is a subgroup $D$ of $E$ isomorphic to $\mathbf{Z} / M \mathbf{Z}$ that lifts $\bar{D}$. Then $(E, C, D)$ gives a point on $\mathcal{M}_{0}(N)\left(\mathcal{O}_{L}\right)$ (cf. [DR73, V.1.6]), whose image in $M_{0}(N)\left(\mathcal{O}_{L}\right)$ corresponds to a point $P$ in $X_{0}(N)\left(\mathcal{O}_{L}\right)$ (since $E$ has ordinary reduction). We will use a bar to denote specialization. Thus we have $Q=\bar{P}$. Similarly, given another point $Q^{\prime} \in C_{0}\left(\overline{\mathbf{F}}_{p}\right)$, we will denote the corresponding associated quantities by a prime superscript (thus $P^{\prime}$ in $X_{0}(N)\left(\mathcal{O}_{L}\right)$ denotes a lift of $Q^{\prime}$, etc.). As mentioned in the previous paragraph, it suffices to prove the relation claimed in the lemma for elements of the form $(Q)-\left(Q^{\prime}\right)$ in $\operatorname{Pic}_{C_{0} / \overline{\mathbf{F}}_{p}}^{0}\left(\overline{\mathbf{F}}_{p}\right)$. Viewing $P$ and $P^{\prime}$ as relative effective Cartier divisors of degree one, we see that $U_{p}\left((Q)-\left(Q^{\prime}\right)\right)$ is the image of $U_{p}\left((P)-\left(P^{\prime}\right)\right)$ under specialization, i.e., $U_{p}\left((Q)-\left(Q^{\prime}\right)\right)=\overline{U_{p}\left((P)-\left(P^{\prime}\right)\right)}$.

We next compute $U_{p}\left((P)-\left(P^{\prime}\right)\right)$. Now $\mathrm{Pic}^{0} X_{0}(N) / \mathcal{O}_{L}$ is the identity component of $J_{0}(N)_{\mathcal{O}_{L}}$, and we have $J_{0}(N)_{\mathcal{O}_{L}}\left(\mathcal{O}_{L}\right)=J_{0}(N)(L) \subseteq J_{0}(N)(\bar{L})$, where 
$\bar{L}$ is an algebraic closure of $L$. Denoting base change to $\bar{L}$ by a subscript $\bar{L}$, we have

$$
\begin{aligned}
& U_{p}\left(\left(E_{\bar{L}}, C_{\bar{L}}, D_{\bar{L}}\right)-\left(E_{\bar{L}}^{\prime}, C_{\bar{L}}^{\prime}, D_{\bar{L}}^{\prime}\right)\right) \\
= & \sum_{A_{\bar{L}}}\left(E_{\bar{L}} / A_{\bar{L}},\left(C_{\bar{L}}+A_{\bar{L}}\right) / A_{\bar{L}},\left(D_{\bar{L}}+A_{\bar{L}}\right) / A_{\bar{L}}\right) \\
- & \sum_{A_{\bar{L}}^{\prime}}\left(E_{\bar{L}}^{\prime} / A_{\bar{L}}^{\prime},\left(C_{\bar{L}}^{\prime}+A_{\bar{L}}^{\prime}\right) / A_{\bar{L}}^{\prime},\left(D_{\bar{L}}^{\prime}+A_{\bar{L}}^{\prime}\right) / A_{\bar{L}}^{\prime}\right),
\end{aligned}
$$

where $A_{\bar{L}}$ runs through the subgroups of $E_{\bar{L}}$ of order $p$ except $C_{\bar{L}}$ (and similarly for $A_{\bar{L}}^{\prime}$ ). Enlarging $L$ by a finite extension if needed (which does not change the residue field $\overline{\mathbf{F}}_{p}$ ) we may assume that there are $p+1$ subgroups of order $p$ in $E_{L}$. Their scheme-theoretic closures in $E$ over $\mathcal{O}_{L}$ are the subgroups schemes mentioned in Lemma 5.14. If $A$ is a subgroup scheme of $E$ of order $p$, then we denote the quotient map $E \rightarrow E / A$ by $\alpha_{A}$. Consider the Cartier divisors corresponding to $U_{p}\left((P)-\left(P^{\prime}\right)\right)$ and to

$$
\begin{gathered}
\left(\pi\left(E / \boldsymbol{\mu}_{p}, \alpha_{\boldsymbol{\mu}_{p}}(C), \alpha_{\boldsymbol{\mu}_{p}}(D)\right)+\sum_{B} \pi\left(E / B, \operatorname{cl}\left(\alpha_{B}(C)\right), \alpha_{B}(D)\right)\right) \\
-\quad\left(\pi\left(E^{\prime} / \boldsymbol{\mu}_{p}^{\prime}, \alpha_{\boldsymbol{\mu}_{p}^{\prime}}\left(C^{\prime}\right), \alpha_{\boldsymbol{\mu}_{p}^{\prime}}\left(D^{\prime}\right)\right)+\sum_{B^{\prime}} \pi\left(E^{\prime} / B^{\prime}, \operatorname{cl}\left(\alpha_{B^{\prime}}\left(C^{\prime}\right)\right), \alpha_{B^{\prime}}\left(D^{\prime}\right)\right)\right),
\end{gathered}
$$

where $B$ runs through the subgroups of $E$ isomorphic to $\mathbf{Z} / p \mathbf{Z}$ except for $C$, and $\operatorname{cl}\left(\alpha_{B}(C)\right)$ denotes the Zariski closure of the image of $B / A$ in $E / A$ (and similarly with prime superscripts). These two divisors coincide since they induce the same $\bar{L}$-point by (4).

Passing to special fibers, and noting that the special fiber of the Néron model of $E / A$ is given by $\bar{E} / \bar{A}$, we find that

$$
\begin{aligned}
& U_{p}\left((Q)-\left(Q^{\prime}\right)\right)=\overline{U_{p}\left((P)-\left(P^{\prime}\right)\right)} \\
= & \left(\left(\bar{E} / \overline{\mu_{p}}, \bar{\alpha}_{\boldsymbol{\mu}_{p}}(\bar{C}), \bar{\alpha}_{\boldsymbol{\mu}_{p}}(\bar{D})\right)+\sum_{B}\left(\bar{E} / \bar{B}, \overline{\operatorname{cl}\left(\alpha_{B}(C)\right)}, \bar{\alpha}_{B}(\bar{D})\right)\right) \\
- & \left(\left(\overline{E^{\prime}} / \overline{\mu_{p}^{\prime}}, \bar{\alpha}_{\boldsymbol{\mu}_{p}^{\prime}}\left(\overline{C^{\prime}}\right), \bar{\alpha}_{\boldsymbol{\mu}_{p}^{\prime}}\left(\overline{D^{\prime}}\right)\right)+\sum_{B^{\prime}}\left(\overline{E^{\prime}} / \overline{B^{\prime}}, \overline{\operatorname{cl}\left(\alpha_{B^{\prime}}\left(C^{\prime}\right)\right)}, \bar{\alpha}_{B^{\prime}}\left(\overline{D^{\prime}}\right)\right)\right),
\end{aligned}
$$

where $B$ again runs through the subgroups of $E$ isomorphic to $\mathbf{Z} / p \mathbf{Z}$ except for $C$ (and a similar statment holds with prime superscripts).

Let $F_{p}$ denote the relative Frobenius map $\bar{E} \rightarrow \bar{E}^{(p)}$ over $\overline{\mathbf{F}}_{p}$. Now $\boldsymbol{\mu}_{p}$ is in the kernel of $F_{p}$, and since the quotient map $\bar{\alpha}_{\boldsymbol{\mu}_{p}}$ has the same degree as $F_{p}$, there is an isomorphism $\phi: \bar{E} / \overline{\mu_{p}} \cong \bar{E}^{(p)}$ such that $F_{p}=\phi \circ \bar{\alpha}_{\boldsymbol{\mu}_{p}}$. Also $\phi$ induces an isomorphism $\bar{\alpha}_{\boldsymbol{\mu}_{p}}(\bar{C}) \stackrel{\cong}{\rightrightarrows} \bar{C}^{(p)}$ and $\bar{\alpha}_{\boldsymbol{\mu}_{p}}(\bar{D}) \stackrel{\cong}{\rightrightarrows} \bar{D}^{(p)}$. Thus the first term in (5) is identified with $\left(\bar{E}^{(p)}, \bar{C}^{(p)}, \bar{D}^{(p)}\right)$, which is the image under Frob $p$ of $\bar{P}=(\bar{E}, \bar{C}, \bar{D})$. Similarly, the first term in $(6)$ is $\operatorname{Frob}_{p}\left(\overline{P^{\prime}}\right)$. 
As for the sum over $B$ in (5), note that in each term, we are quotienting by a group $B$ which is isomorphic to $\mathbf{Z} / p \mathbf{Z}$, and hence $\operatorname{cl}\left(\alpha_{B}(C)\right)$ is of $\boldsymbol{\mu}_{p}$-type. In a manner similar to the computation of the action of $U_{p}$, we find that

$$
\begin{aligned}
& W_{p}\left((\bar{E}, \bar{C}, \bar{D})-\left(\overline{E^{\prime}}, \overline{C^{\prime}}, \overline{D^{\prime}}\right)\right) \\
= & (\bar{E} / \bar{C}, \bar{E}[p] / \bar{C},(\bar{D}+\bar{C}) / \bar{C}) \\
- & \left(\overline{E^{\prime}} / \overline{C^{\prime}}, \overline{E^{\prime}}[p] / \overline{C^{\prime}},\left(\overline{D^{\prime}}+\overline{C^{\prime}}\right) / \overline{C^{\prime}}\right) .
\end{aligned}
$$

Considering that $\bar{P}=(\bar{E}, \bar{C}, \bar{D})$, with $\bar{C}$ isomorphic to $\mathbf{Z} / p \mathbf{Z}$, we see that $\bar{E}[p] / \bar{C}$ is isomorphic to $\boldsymbol{\mu}_{p}$. Also, if $B$ is as in the sum in (5), then $\bar{B}$ is a $\mathbf{Z} / p \mathbf{Z}$, but there is only one copy of $\mathbf{Z} / p \mathbf{Z}$ in $\bar{E}$, since $E$ has good ordinary reduction; hence $\bar{B}=\bar{C}$. Thus each of the terms in the sum over $B$ in (5) is the term in (7). A similar statment holds with prime superscripts (viz., each of the terms in the sum over $B^{\prime}$ in (6) is the term in (8)).

The lemma now follows from the previous two paragraphs.

Since we are assuming that $p \| N$, the curve $X_{0}(N)_{\overline{\mathbf{F}}_{p}}$ has ordinary double point singularities, and so the differentials in $H^{0}\left(X_{0}(N)_{\overline{\mathbf{F}}_{p}}, \Omega_{X_{0}(N) / \overline{\mathbf{F}}_{p}}\right)$ may be identified with meromorphic differentials $\left(\omega_{i}\right)_{i=0, \ldots, r}$ on $\prod_{i=0}^{r} C_{i}$ whose only possible poles are at points on $\prod_{i=0}^{r} C_{i}$ lying over an intersection point of two components in $X_{0}(N)_{\overline{\mathbf{F}}_{p}}$ and where the sum of the residues at the points lying over an intersection point is zero; such differentials are called regular differentials (see [Con00, §5.2] for the justification that the relative dualizing sheaf under Grothendieck duality is indeed the sheaf of regular differentials). By a holomorphic differential in $H^{0}\left(X_{0}(N)_{\overline{\mathbf{F}}_{p}}, \Omega_{X_{0}(N) / \overline{\mathbf{F}}_{p}}\right)$, we mean a regular differential all of whose corresponding $\omega_{i}$ have no poles at all (i.e., for all $\left.i, \omega_{i} \in H^{0}\left(C_{i}, \Omega_{C_{i}} / \overline{\mathbf{F}}_{p}\right)\right)$. The subspace of holomorphic differentials may be identified with $\prod_{i=0}^{r} H^{0}\left(C_{i}, \Omega_{C_{i} / \overline{\mathbf{F}}_{p}}\right.$ ) (which we will often do implicitly), and we let $i_{1}$ denote the corresponding injection $\prod_{i=0}^{r} H^{0}\left(C_{i}, \Omega_{C_{i} / \overline{\mathbf{F}}_{p}}\right) \hookrightarrow$ $H^{0}\left(X_{0}(N) \overline{\mathbf{F}}_{p}, \Omega_{X_{0}(N) / \overline{\mathbf{F}}_{p}}\right)$.

In a manner similar to the description in the third paragraph of Section 5.2, Grothendieck duality gives an isomorphism

$$
\Theta: H^{0}\left(X_{0}(N)_{\mathcal{O}_{L}}, \Omega_{X_{0}(N) / \mathcal{O}_{L}}\right) \stackrel{\cong}{\rightrightarrows} \operatorname{Cot}\left(\operatorname{Pic}_{X_{0}(N) / \mathcal{O}_{L}}^{0}\right),
$$

where Cot denotes the cotangent space at the identity section. Since we have an action of $U_{p}$ and $W_{p}$ on $\operatorname{Pic}_{X_{0}(N) / \mathcal{O}_{L}}^{0}$ (by viewing it as the identity component of the Néron model of $J_{0}(N)$ over $\left.\mathcal{O}_{L}\right)$, we may use $\Theta$ to get an action of these operators on $H^{0}\left(X_{0}(N)_{\mathcal{O}_{L}}, \Omega_{X_{0}(N) / \mathcal{O}_{L}}\right)$. As before, Prop. 3.3 on p. 68 of [Maz77] implies that base change to $\overline{\mathbf{F}}_{p}$ gives an isomorphism

$$
\mathrm{H}^{0}\left(X_{0}(N)_{\overline{\mathbf{F}}_{p}}, \Omega_{X_{0}(N) / \overline{\mathbf{F}}_{p}}\right) \cong \mathrm{H}^{0}\left(X_{0}(N)_{\mathcal{O}_{L}}, \Omega_{X_{0}(N) / \mathcal{O}_{L}}\right) \otimes_{\mathcal{O}_{L}} \overline{\mathbf{F}}_{p}
$$

From this, we get an action of $U_{p}$ and $W_{p}$ on $H^{0}\left(X_{0}(N)_{\overline{\mathbf{F}}_{p}}, \Omega_{X_{0}(N) / \overline{\mathbf{F}}_{p}}\right)$. 
COROLlaRY 5.16. The endomorphisms $U_{p}$ and $W_{p}$ of $\mathrm{H}^{0}\left(X_{0}(N)_{\overline{\mathbf{F}}_{p}}, \Omega_{X_{0}(N) / \overline{\mathbf{F}}_{p}}\right)$ preserve the subspace $\prod_{i=0}^{r} H^{0}\left(C_{i}, \Omega_{C_{i} / \overline{\mathbf{F}}_{p}}\right)$, and satisfy $U_{p}= \pm$ Frob $_{p}^{*}+(p-$ 1) $W_{p}$ on $H^{0}\left(C_{0}, \Omega_{C_{0} / \overline{\mathbf{F}}_{p}}\right)$, where Frob Fr $_{p}^{*}$ denotes pullback by Frob Fnd $_{p}$ and where we have a possible sign ambiguity \pm (which will not affect us later).

Proof. The proof is based on the following diagram; we describe below some of the maps in it that have not been defined yet.

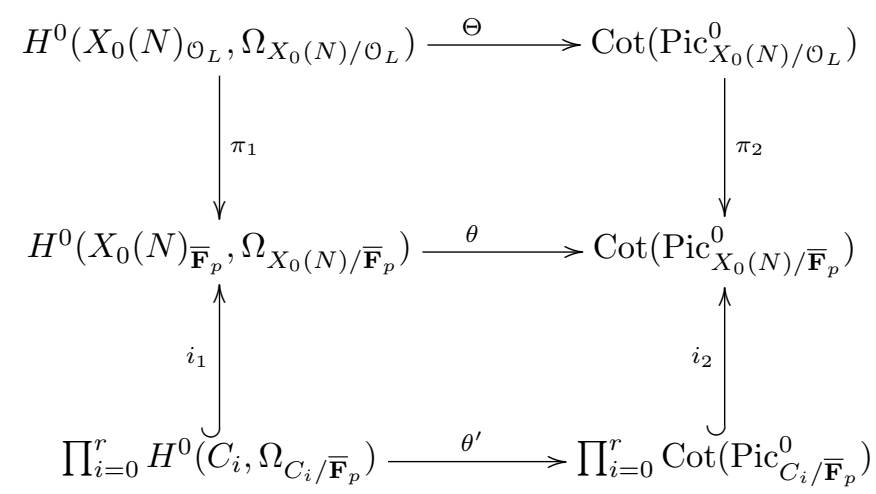

Firstly, Cot always denotes the cotangent space at the identity section. The map $\pi_{1}$ is obtained by base change to $\overline{\mathbf{F}}_{p}$. By (10), $\pi_{1}$ is surjective. The map $\pi_{2}$ is obtained by observing that $\mathrm{Pic}_{X_{0}(N) / \overline{\mathbf{F}}_{p}}^{0}$ is the identity component of the special fiber of the Néron model of $J_{0}(N)$ over $\mathcal{O}_{L}$, and hence maps to the identity component of the Néron model of $J_{0}(N)$ over $\mathcal{O}_{L}$, which is $\operatorname{Pic}^{0}{ }_{X_{0}}(N) / \mathcal{O}_{L}$. The map $\theta$ is obtained using Grothendieck duality. The compatibility of Grothendieck duality under base change (see [Con00]) implies that the top square in the diagram above commutes.

Now we have already defined actions of $U_{p}$ and $W_{p}$ on $\operatorname{Pic}_{X_{0}(N) / \mathcal{O}_{L}}^{0}$ and on $\mathrm{Pic}_{X_{0}(N) / \overline{\mathbf{F}}_{p}}^{0}$ (just before Lemma 5.15). Thus we get actions of $U_{p}$ and $W_{p}$ on $\operatorname{Cot}\left(\operatorname{Pic}_{X_{0}(N) / \mathcal{O}_{L}}^{0}\right)$ and on $\operatorname{Cot}\left(\operatorname{Pic}_{X_{0}(N) / \overline{\mathbf{F}}_{p}}^{0}\right)$. From the definitions of these actions we see that $\pi_{2}$ is compatible with the actions on its domain and codomain. Recall that we used the isomorphism $\Theta$ to induce actions of $U_{p}$ and $W_{p}$ on $H^{0}\left(X_{0}(N)_{\mathcal{O}_{L}}, \Omega_{X_{0}(N) / \mathcal{O}_{L}}\right)$ and then used formula (10) to get actions on $H^{0}\left(X_{0}(N)_{\overline{\mathbf{F}}_{p}}, \Omega_{X_{0}(N) / \overline{\mathbf{F}}_{p}}\right)$. Thus $\Theta$ and $\pi_{1}$ are also compatible with with the actions of $U_{p}$ and $W_{p}$ on their domain and codomain. Let $\omega \in H^{0}\left(X_{0}(N)_{\overline{\mathbf{F}}_{p}}, \Omega_{X_{0}(N) / \overline{\mathbf{F}}_{p}}\right)$, and let $\Omega \in H^{0}\left(X_{0}(N)_{\mathcal{O}_{L}}, \Omega_{X_{0}(N) / \mathcal{O}_{L}}\right)$ be such that $\pi_{1}(\Omega)=\omega$. Then $\theta\left(U_{p}(\omega)\right)=\theta\left(\pi_{1}\left(U_{p}(\Omega)\right)\right)=\pi_{2}\left(\Theta\left(U_{p}(\Omega)\right)\right)=$ $\pi_{2}\left(U_{p}(\Theta(\Omega))\right)=U_{p}\left(\pi_{2}(\Theta(\Omega))\right)=U_{p}\left(\theta\left(\pi_{2}(\Omega)\right)\right)=U_{p}(\theta(\omega))$. Thus we see that the isomorphism $\theta$ is compatible with the action of $U_{p}$ (and similarly for $W_{p}$ ) on its domain and codomain.

Now we turn to the bottom square in the diagram above. As mentioned earlier, the injection $i_{2}$ arises because $\prod_{i=0}^{r} \operatorname{Pic}_{C_{i} / \overline{\mathbf{F}}_{p}}^{0}$ is the maximal abelian variety quotient of the identity component $\mathrm{Pic}_{X_{0}(N) / \overline{\mathbf{F}}_{p}}^{0}$ of the special fiber of 
the Néron model of $J_{0}(N)$ over $\mathcal{O}_{L}$. The map $\theta^{\prime}$ is the isomorphism coming from Serre duality.

Next, by [Con00, $\S 5.2]$, the Grothendieck duality isomorphism $\theta$ is the same as the isomorphism coming from the duality theory of Rosenlicht (as in [Ser88, Chap. IV]), perhaps up to multiplication by -1 . Assume for the moment that there is no sign ambiguity, so that $\theta$ is indeed the isomorphism coming from the duality theory of Rosenlicht. One can check that the Serre duality isomorphism $\theta^{\prime}$ is induced by the Rosenlicht duality isomorphism $\theta$ via the inclusions $i_{1}$ and $i_{2}$ by looking at the proof of the two dualities in [Ser88, Chaps. II and IV]. Note that in [Ser88], the curve $X$ over the field $k$ (notation as in loc. cit.) is assumed to be irreducible. This hypothesis is needed in loc. cit. (for our purposes) only to show that $H^{1}(X, k(X))=0$ (p. 12, loc. cit.); the latter condition holds so long as $X$ is reduced (see top of p. 165 in [AK70], as well as the bottom of p. 138 and top of p. 132 therein), which is true in our case (taking $X=X_{0}(N)_{\overline{\mathbf{F}}_{p}}$ and $k=\overline{\mathbf{F}}_{p}$ ). We remark that our contention that the Serre duality isomorphism $\theta^{\prime}$ is induced by the Rosenlicht duality isomorphism $\theta$ via the inclusions $i_{1}$ and $i_{2}$ also follows from Section 6 (an appendix provided to us by Brian Conrad), by taking $C=X_{0}(N)_{\overline{\mathbf{F}}_{p}}$ and $C^{\prime}$ to be any of the $C_{i}$ in Section 6 . In any case, we conclude that the bottom square in the diagram above commutes as well, perhaps up to multiplication by -1 .

Now the action of $U_{p}$ and $W_{p}$ on $\prod_{i=0}^{r} \operatorname{Pic}_{C_{i} / \overline{\mathbf{F}}_{p}}^{0}$ was defined by identifying it as the maximal abelian variety quotient of $\operatorname{Pic}_{X_{0}(N) / \overline{\mathbf{F}}_{p}}^{0}$. Thus we see that $i_{2}$ is compatible with the action of $U_{p}$ and $W_{p}$ on its domain and codomain. Considering that moreover the isomorphism $\theta$ is compatible with the action of $U_{p}$ (and $W_{p}$ ) and the bottom square in the diagram above commutes, perhaps up to multiplication by -1 , we see that $U_{p}$ and $W_{p}$ preserve $\prod_{i=0}^{r} H^{0}\left(C_{i}, \Omega_{C_{i}} / \overline{\mathbf{F}}_{p}\right)$. Now since $\theta$ is compatible with the action of $U_{p}$ and $W_{p}$ on its domain and codomain, so is $\theta^{\prime}$. Thus we may use the isomorphism $\theta^{\prime}$ to translate the identity in Lemma 5.15 from the right to the left of $\theta^{\prime}$ to get the desired identity in the Corollary, where the \pm ambiguity in front of $\mathrm{Frob}_{p}{ }^{*}$ is really due to the sign ambiguity about the compatibility of the action of $U_{p}$ and $W_{p}$ on the two sides of the isomorphism $\theta^{\prime}$.

REMark 5.17. We defined the action of the Hecke operators and the AtkinLehner involution in characteristic $p$ from their definition in characteristic 0 in a somewhat indirect manner via the Néron mapping property, Grothendieck duality, etc (cf. beginning of Section 5.2). This has made our proofs rather complicated, since we have to show several compatibilities (as in the previous Corollary 5.16 and the upcoming Lemma 5.18). After this article was written, B. Conrad pointed out to us that one can define the action of the Hecke operators on suitable Artin stacks over $\mathbf{Z}$ for $\Gamma_{0}(N)$-structures (see [Con07]) in such a way that the definition agrees with the usual definition of the Hecke operators over $\mathbf{Q}$. This naturally defines the action of the Hecke operators on objects 
related to $X_{0}(N)$ such as differentials, Picard groups, etc., in characteristic $p$ and these definitions are automatically "compatible" with the corresponding definitions in characteristic zero. This alternative method would have been a less complicated way to proceed.

By [Maz77, Prop. II.3.3] we have an isomorphism

$$
\mathrm{H}^{0}\left(X_{0}(N)_{\overline{\mathbf{F}}_{p}}, \Omega_{X_{0}(N) / \overline{\mathbf{F}}_{p}}\right) \cong \mathrm{H}^{0}\left(X_{0}(N)_{\mathbf{F}_{p}}, \Omega_{X_{0}(N) / \mathbf{F}_{p}}\right) \otimes_{\mathbf{F}_{p}} \overline{\mathbf{F}}_{p},
$$

using which we may identify $\mathrm{H}^{0}\left(X_{0}(N)_{\mathbf{F}_{p}}, \Omega_{X_{0}(N) / \mathbf{F}_{p}}\right)$ as a subspace of $\mathrm{H}^{0}\left(X_{0}(N)_{\overline{\mathbf{F}}_{p}}, \Omega_{X_{0}(N) / \overline{\mathbf{F}}_{p}}\right)$. Just before Corollary 5.16 , we defined an action of $U_{p}$ (and $\left.W_{p}\right)$ on $\mathrm{H}^{0}\left(X_{0}(N)_{\overline{\mathbf{F}}_{p}}, \Omega_{X_{0}(N) / \overline{\mathbf{F}}_{p}}\right)$.

LEMmA 5.18. The action of $U_{p}$ (respectively $\left.W_{p}\right)$ on $\mathrm{H}^{0}\left(X_{0}(N)_{\overline{\mathbf{F}}_{p}}, \Omega_{X_{0}(N) / \overline{\mathbf{F}}_{p}}\right)$ preserves the subspace $\mathrm{H}^{0}\left(X_{0}(N)_{\mathbf{F}_{p}}, \Omega_{X_{0}(N) / \mathbf{F}_{p}}\right)$, and agrees with the action of $U_{p}$ (respectively $W_{p}$ ) on this subspace that we defined earlier in the third paragraph of Section 5.2.

Proof. We have the following diagram, obtained by the obvious base changes:

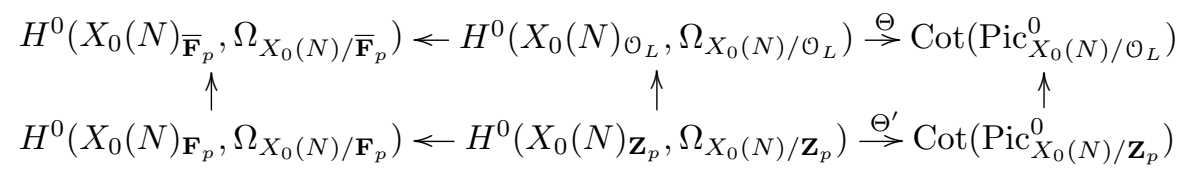

where the map $\Theta^{\prime}$ is the isomorphism coming from Grothendieck duality as discussed in the third paragraph of Section 5.2. Now the action of $U_{p}$ and $W_{p}$ on $\operatorname{Cot}\left(\operatorname{Pic}_{X_{0}(N) / \mathcal{O}_{L}}^{0}\right)=\operatorname{Cot}\left(J_{0}(N)_{\mathcal{O}_{L}}\right)$ (where $J_{0}(N)_{\mathcal{O}_{L}}$ is the Néron model of $J_{0}(N)$ over $\mathcal{O}_{L}$ ) was obtained by base changing from $\mathbf{Z}_{p}$. Considering that the formation of Néron models is compatible with completions and unramified base change, we see that the rightmost vertical map above is compatible under the action of $U_{p}$ and $W_{p}$. Also, the action of $U_{p}$ and $W_{p}$ on $H^{0}\left(X_{0}(N)_{\mathcal{O}_{L}}, \Omega_{X_{0}(N) / \mathcal{O}_{L}}\right)$ (respectively on $H^{0}\left(X_{0}(N)_{\overline{\mathbf{F}}_{p}}, \Omega_{X_{0}(N) / \overline{\mathbf{F}}_{p}}\right)$ ) was obtained via the isomorphism $\Theta$ (respectively $\Theta^{\prime}$ ). Thus the rightmost two horizonal maps above are also compatible under the action of $U_{p}$ and $W_{p}$ on their domain and codomain. Finally, the compatibility of Grothendieck duality under base change (see [Con00]) implies that the right square in the diagram above commutes. Arguing as in the third paragraph of the proof of Corollary 5.16, one sees then that the middle vertical map above is compatible under the action of $U_{p}$ and $W_{p}$.

Now the already defined action of $U_{p}$ and $W_{p}$ on $\mathrm{H}^{0}\left(X_{0}(N)_{\mathbf{F}_{p}}, \Omega_{X_{0}(N) / \mathbf{F}_{p}}\right)$ in the third paragraph of Section 5.2 is obtained via the lower leftward pointing arrow in the diagram above, and the action of $U_{p}$ and $W_{p}$ on $\mathrm{H}^{0}\left(X_{0}(N)_{\overline{\mathbf{F}}_{p}}, \Omega_{X_{0}(N) / \overline{\mathbf{F}}_{p}}\right)$ is obtained via the upper leftward pointing arrow in the diagram above. Thus the leftmost two horizontal arrows are compatible under the action of $U_{p}$ and $W_{p}$ on their domain and codomain. Repeated applications of [Maz77, Prop. II.3.3] show that the left square also 
commutes. Using all this, we see that the the action of $U_{p}$ (respectively $W_{p}$ ) on $\mathrm{H}^{0}\left(X_{0}(N)_{\mathbf{F}_{p}}, \Omega_{X_{0}(N) / \mathbf{F}_{p}}\right)$ viewed as a subspace of $\mathrm{H}^{0}\left(X_{0}(N)_{\overline{\mathbf{F}}_{p}}, \Omega_{X_{0}(N) / \overline{\mathbf{F}}_{p}}\right)$ agrees with the action of $U_{p}$ (respectively $\left.W_{p}\right)$ on $\mathrm{H}^{0}\left(X_{0}(N) \mathbf{F}_{p}, \Omega_{X_{0}(N) / \mathbf{F}_{p}}\right)$ defined in the third paragraph of Section 5.2, and in particular that $U_{p}$ and $W_{p}$ preserve this subspace.

We now revert to the assumption that $p$ is a prime such that $p^{2} \nmid N$ (in particular $p$ may not necessarily divide $N)$. The Tate curve over $\mathbf{F}_{p}[[q]]$ gives rise to a morphism from Spec $\mathbf{F}_{p}[[q]]$ to the smooth locus of $X_{0}(N) \mathbf{F}_{p} \rightarrow \operatorname{Spec} \mathbf{F}_{p}$. Since the module of completed Kahler differentials for $\mathbf{F}_{p}[[q]]$ over $\mathbf{F}_{p}$ is free of rank 1 on the basis $d q$, we obtain a map

$$
q-\exp : H^{0}\left(X_{0}(N)_{\mathbf{F}_{p}}, \Omega_{X_{0}(N) / \mathbf{F}_{p}}\right) \rightarrow \mathbf{F}_{p}[[q]]
$$

If $p \nmid N$, then by a holomorphic differential in $H^{0}\left(X_{0}(N)_{\mathbf{F}_{p}}, \Omega_{X_{0}(N) / \mathbf{F}_{p}}\right)$, we mean any differential in $H^{0}\left(X_{0}(N)_{\mathbf{F}_{p}}, \Omega_{X_{0}(N) / \mathbf{F}_{p}}\right)$.

LEMMA 5.19. Recall that $p$ is a prime such that $p^{2} \nmid N$, and $\mathfrak{m}$ is a maximal ideal of $\mathbf{T}$ with residue characteristic $p$. If $p \mid N$, then assume that $U_{p}$ acts as a non-zero scalar on $H^{0}\left(X_{0}(N)_{\mathbf{F}_{p}}, \Omega_{X_{0}(N) / \mathbf{F}_{p}}\right)[\mathfrak{m}]$. Then the map q-exp restricted to homomorphic differentials in $\mathrm{H}^{0}\left(X_{0}(N) \mathbf{F}_{p}, \Omega_{X_{0}(N) / \mathbf{F}_{p}}\right)[\mathfrak{m}]$ is injective.

Proof. The essential argument is quite standard, going back to Mazur, so we only sketch the ideas. For some of the details, we refer the reader to the proof of Lemma 4.2 in [ARS06]. If $p \nmid N$, the injectivity follows from the $q$-expansion principle. So suppose that $p \| N$, and let $M=N / p$. Recall that $X_{0}(N)_{\overline{\mathbf{F}}_{p}}$ is obtained from $M_{0}(N)_{\overline{\mathbf{F}}_{p}}$ by suitable blowups at supersingular points and consists of two copies of $X_{0}(M)_{\overline{\mathbf{F}}_{p}}$ identified at supersingular points, along with some copies of $\mathbf{P}^{1}$. Suppose $\omega \in H^{0}\left(X_{0}(N)_{\mathbf{F}_{p}}, \Omega_{X_{0}(N) / \mathbf{F}_{p}}\right)[\mathfrak{m}]$ is a holomorphic differential that is in the kernel of $q$-exp. Then the $q$-expansion principle implies that $\omega$ vanishes on the copy of $X_{0}(M)_{\overline{\mathbf{F}}_{p}}$ containing the cusp $\infty$, i.e., on $C_{1}$. By Corollary 5.16 and Lemma 5.18, we have $U_{p}\left(\left.\omega\right|_{C_{0}}\right)= \pm \operatorname{Frob}_{p}{ }^{*}\left(\left.\omega\right|_{C_{0}}\right)+(p-$

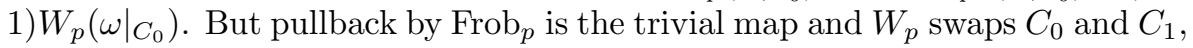
so $U_{p}\left(\left.\omega\right|_{C_{0}}\right)=(p-1)\left(\left.\omega\right|_{C_{1}}\right)=0$. Now by hypothesis, $U_{p}$ acts as multiplication by a non-zero scalar, hence $\omega$ is trivial on $C_{0}$. Thus $\omega$ is trivial on both copies of $X_{0}(M)_{\mathbf{F}_{p}}$. One can show that then $\omega$ is trivial on the copies of $\mathbf{P}^{1}$ as well (see the proof of Lemma 4.2 in [ARS06]). Thus $\omega$ is trivial on $X_{0}(N)_{\overline{\mathbf{F}}_{p}}$, hence on $X_{0}(N) \mathbf{F}_{p}$.

LEMMA 5.20. We continue our hypotheses that $p$ is a prime such that $p^{2} \nmid N$, $\mathfrak{m}$ is a maximal ideal of $\mathbf{T}$ with residue characteristic $p$, and if $p \mid N$, then $U_{p}$ acts as a non-zero scalar on $H^{0}\left(X_{0}(N) \mathbf{F}_{p}, \Omega_{X_{0}(N) / \mathbf{F}_{p}}\right)[\mathfrak{m}]$. Then

$$
\operatorname{dim}_{\mathbf{T} / \mathfrak{m}} \mathrm{H}^{0}\left(X_{0}(N)_{\mathbf{F}_{p}}, \Omega_{X_{0}(N) / \mathbf{F}_{p}}\right)[\mathfrak{m}] \leq 1
$$


Proof. The idea behind the proof is the same as in the proof of Lemma 2.2 in [Wil80, p. 485-487], which in turn builds on ideas from p. 94-95 of [Maz77]. However, parts of our arguments are somewhat different, and may be considered alternatives to some of the methods in the works cited in the previous sentence.

If $\omega \in \mathrm{H}^{0}\left(X_{0}(N)_{\mathbf{F}_{p}}, \Omega_{X_{0}(N) / \mathbf{F}_{p}}\right)$ and $n \geq 1$, then let $a_{n}(\omega)$ denote the coefficient of $q^{n}$ in $q-\exp (\omega)$. We have a pairing $\mathrm{H}^{0}\left(X_{0}(N)_{\mathbf{F}_{p}}, \Omega_{X_{0}(N) / \mathbf{F}_{p}}\right) \times \mathbf{T} \rightarrow$ $\mathbf{F}_{p}$ that takes $(\omega, T)$ to $a_{1}(T \omega)$. This induces a map

$$
\psi: \mathrm{H}^{0}\left(X_{0}(N)_{\mathbf{F}_{p}}, \Omega_{X_{0}(N) / \mathbf{F}_{p}}\right)[\mathfrak{m}] \rightarrow \operatorname{Hom}_{\mathbf{F}_{p}}\left(\mathbf{T} / \mathfrak{m}, \mathbf{F}_{p}\right),
$$

which is a homomorphism of $\mathbf{T} / \mathfrak{m}$-vector spaces.

Claim 1: If $\omega \in \operatorname{ker}(\psi)$, then $q$-exp $(\omega)$ is trivial.

Proof. Following the proof of Prop. 3.3 on p. 68 of [Maz77], we have

$$
\mathrm{H}^{0}\left(X_{0}(N)_{\mathbf{F}_{p}}, \Omega_{X_{0}(N) / \mathbf{F}_{p}} \cong \mathrm{H}^{0}\left(X_{0}(N) \mathbf{z}_{p}, \Omega_{X_{0}(N) / \mathbf{z}_{p}}\right) \otimes \mathbf{z}_{p} \mathbf{F}_{p},\right.
$$

and

$$
\mathrm{H}^{0}\left(X_{0}(N)(\mathbf{C}), \Omega_{X_{0}(N)(\mathbf{C}) / \mathbf{C}}\right) \cong \mathrm{H}^{0}\left(X_{0}(N) \mathbf{z}_{p}, \Omega_{X_{0}(N) / \mathbf{z}_{p}}\right) \otimes_{\mathbf{z}_{p}} \mathbf{C} .
$$

The definition of the action of the Hecke operators on $\mathrm{H}^{0}\left(X_{0}(N) \mathbf{z}_{p}, \Omega_{X_{0}(N) / \mathbf{z}_{p}}\right)$ defined in the third paragraph of Section 5.2 shows that this action is compatible with the action of the Hecke operators on $\mathrm{H}^{0}\left(X_{0}(N)(\mathbf{C}), \Omega_{\left.X_{0}(N)(\mathbf{C}) / \mathbf{C}\right)}\right.$ under (12). Also, the action of the Hecke operators on $\mathrm{H}^{0}\left(X_{0}(N)_{\mathbf{F}_{p}}, \Omega_{X_{0}(N) / \mathbf{F}_{p}}\right)$ was defined in the third paragraph of Section 5.2 via their action on $\mathrm{H}^{0}\left(X_{0}(N) \mathbf{z}_{p}, \Omega_{X_{0}(N) / \mathbf{z}_{p}}\right)$ using (11), so these actions are clearly compatible under (11). Now

$$
\mathrm{H}^{0}\left(X_{0}(N)(\mathbf{C}), \Omega_{X_{0}(N)(\mathbf{C}) / \mathbf{C}}\right) \cong \mathrm{H}^{0}\left(J_{0}(N)(\mathbf{C}), \Omega_{J_{0}(N)(\mathbf{C}) / \mathbf{C}}\right) \cong S_{2}\left(\Gamma_{0}(N), \mathbf{C}\right),
$$

and thus $a_{1}\left(T_{n} \omega\right)=a_{n}(\omega)$ for $\omega \in \mathrm{H}^{0}\left(X_{0}(N)(\mathbf{C}), \Omega_{\left.X_{0}(N)(\mathbf{C}) / \mathbf{C}\right) \text {. Hence, }}\right.$ by (11), (12), and the discussion above, we also have the formula $a_{1}\left(T_{n} \omega\right)=$ $a_{n}(\omega)$ for $\omega \in \mathrm{H}^{0}\left(X_{0}(N)_{\mathbf{F}_{p}}, \Omega_{X_{0}(N) / \mathbf{F}_{p}}\right)$.

Thus if $\omega \in \operatorname{ker}(\psi)$, then $a_{n}(\omega)=a_{1}\left(T_{n} \omega\right)=0$ for all $n \geq 1$, i.e., $q$-exp $(\omega)$ is trivial, as was to be shown.

Claim 2: The $\mathbf{T} / \mathfrak{m}$-dimension of the subspace of holomorphic differentials in $\mathrm{H}^{0}\left(X_{0}(N)_{\mathbf{F}_{p}}, \Omega_{X_{0}(N) / \mathbf{F}_{p}}\right)[\mathfrak{m}]$ is at most 1 .

Proof. If $\omega$ is a holomorphic differential in $\mathrm{H}^{0}\left(X_{0}(N)_{\mathbf{F}_{p}}, \Omega_{X_{0}(N) / \mathbf{F}_{p}}\right)[\mathfrak{m}]$ and $\psi(\omega)=0$, then by Claim 1, $q$-exp $(\omega)$ is trivial, and hence by Lemma $5.19, \omega$ is trivial. This proves that $\psi$ is injective when restricted to the subspace of holomorphic differentials. Now the group $\operatorname{Hom}_{\mathbf{F}_{p}}\left(\mathbf{T} / \mathfrak{m}, \mathbf{F}_{p}\right)$ has the same size as $\mathbf{T} / \mathfrak{m}$, which completes the argument because $\psi$ embeds the subspace of holomorphic differentials in $\mathrm{H}^{0}\left(X_{0}(N)_{\mathbf{F}_{p}}, \Omega_{X_{0}(N) / \mathbf{F}_{p}}\right)[\mathfrak{m}]$ into $\operatorname{Hom}_{\mathbf{F}_{p}}\left(\mathbf{T} / \mathfrak{m}, \mathbf{F}_{p}\right)$, which has dimension 1 as a $\mathbf{T} / \mathfrak{m}$-vector space. 
Claim 2 proves the lemma in the case when $p \nmid N$. We now prove that $\operatorname{dim}_{\mathbf{T} / \mathfrak{m}} \mathrm{H}^{0}\left(X_{0}(N)_{\mathbf{F}_{p}}, \Omega_{X_{0}(N) / \mathbf{F}_{p}}\right)[\mathfrak{m}] \leq 1$ when $p \| N$, which will finish the proof of the lemma. Following the proof of Lemma 2.2 in [Wil95], we break the argument into two cases:

Case I: There is no nonzero holomorphic differential in

$$
\mathrm{H}^{0}\left(X_{0}(N) \mathbf{F}_{p}, \Omega_{X_{0}(N) / \mathbf{F}_{p}}\right)[\mathfrak{m}]
$$

Suppose $\omega_{1}$ and $\omega_{2}$ are two differentials in $\mathrm{H}^{0}\left(X_{0}(N)_{\mathbf{F}_{p}}, \Omega_{X_{0}(N) / \mathbf{F}_{p}}\right)[\mathfrak{m}]$. Then we can find a pair $(\mu, \lambda) \in(\mathbf{T} / \mathfrak{m})^{2}$ with $(\mu, \lambda) \neq(0,0)$ such that $\mu \psi\left(\omega_{1}\right)-\lambda \psi\left(\omega_{2}\right)=0$, i.e., $\psi\left(\mu \omega_{1}-\lambda \omega_{2}\right)=0$. Hence by Claim 1, $q-\exp \left(\mu \omega_{1}-\lambda \omega_{2}\right)=0$. Viewing $\mu \omega_{1}-\lambda \omega_{2}$ as an element

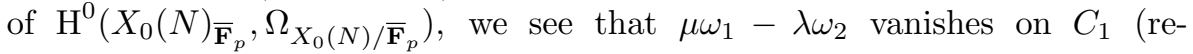
call that $C_{1}$ is the copy of $X_{0}(N / p)_{\overline{\mathbf{F}}_{p}}$ that contains the cusp $\left.\infty\right)$ by the "q-expansion principle" (see the proof of Lemma 4.2 in [ARS06] for details). Now $C_{2}, \ldots, C_{r}$ (the copies of $\mathbf{P}^{1}$ ) arise as chains that link $C_{1}$ and $C_{0}$ (recall that $C_{0}$ is the copy of $X_{0}(N / p)_{\overline{\mathbf{F}}_{p}}$ that does not contain the cusp $\left.\infty\right)$ and each of $C_{2}, \ldots, C_{r}$ has at most two points of intersection, with all intersection points being ordinary double points (see the description of $X_{0}(N)_{\overline{\mathbf{F}}_{p}}$ on p. 175-177 of [Maz77] for details). Taking into consideration the definition of regular differentials and the residue theorem we see that $\mu \omega_{1}-\lambda \omega_{2}$ is holomorphic on the curves among $C_{2}, \ldots, C_{r}$ that intersect $C_{1}$ (for details, see the proof of Lemma 4.2 in [ARS06] in a similar situation). Now a curve among $C_{2}, \ldots, C_{r}$ that does not intersect $C_{1}$ intersects exactly one curve among $C_{2}, \ldots, C_{r}$ that does intersect $C_{1}$. Hence by repeating the argument above, $\mu \omega_{1}-\lambda \omega_{2}$ is holomorphic on each curve in $C_{2}, \ldots, C_{r}$ that does not intersect $C_{1}$ as well. Thus $\mu \omega_{1}-\lambda \omega_{2}$ is holomorphic on all of $X_{0}(N)_{\overline{\mathbf{F}}_{p}}$ except perhaps on $C_{0}$. But the only possible poles of $\mu \omega_{1}-\lambda \omega_{2}$ on $C_{0}$ are over points of intersection with other components, and again, considering the definition of regular differentials, we see that there are no such poles, i.e., $\mu \omega_{1}-\lambda \omega_{2}$ is holomorphic on $C_{0}$ as well. Thus $\mu \omega_{1}-\lambda \omega_{2}$ is holomorphic everywhere and is an element of $\mathrm{H}^{0}\left(X_{0}(N)_{\mathbf{F}_{p}}, \Omega_{X_{0}(N) / \mathbf{F}_{p}}\right)[\mathfrak{m}]$. Hence it is trivial by the hypothesis of this case. Thus $\omega_{1}$ and $\omega_{2}$ are linearly dependent. Since $\omega_{1}$ and $\omega_{2}$ were arbitrary, this shows that $\operatorname{dim}_{\mathbf{T} / \mathfrak{m}} \mathrm{H}^{0}\left(X_{0}(N) \mathbf{F}_{p}, \Omega_{X_{0}(N) / \mathbf{F}_{p}}\right)[\mathfrak{m}] \leq 1$ in this case.

Case II: There is a nonzero holomorphic differential

$$
\omega \in \mathrm{H}^{0}\left(X_{0}(N)_{\mathbf{F}_{p}}, \Omega_{X_{0}(N) / \mathbf{F}_{p}}\right)[\mathfrak{m}] .
$$

By Lemma 5.19, $q$-exp $(\omega)$ is non-trivial, and so by Claim $1, \psi(\omega) \neq 0$. Let $\omega^{\prime} \in \mathrm{H}^{0}\left(X_{0}(N)_{\mathbf{F}_{p}}, \Omega_{X_{0}(N) / \mathbf{F}_{p}}\right)[\mathfrak{m}]$. Then there is a $\lambda \in \mathbf{T} / \mathfrak{m}$ such that $\psi\left(\omega^{\prime}\right)-$ $\lambda \psi(\omega)=0$, i.e., $\psi\left(\omega^{\prime}-\lambda \omega\right)=0$. As in the proof of Case I, we conclude that $\omega^{\prime}-\lambda \omega$ is holomorphic; in particular $\omega^{\prime}$ is holomorphic. Thus every differential in $\mathrm{H}^{0}\left(X_{0}(N) \mathbf{F}_{p}, \Omega_{X_{0}(N) / \mathbf{F}_{p}}\right)[\mathfrak{m}]$ is holomorphic. Then by Claim 2, $\operatorname{dim}_{\mathbf{T} / \mathfrak{m}} \mathrm{H}^{0}\left(X_{0}(N) \mathbf{F}_{p}, \Omega_{X_{0}(N) / \mathbf{F}_{p}}\right)[\mathfrak{m}] \leq 1$ in this case as well. 
Proof of Proposition 5.10. Recall that the hypotheses of Proposition 5.10 are that $p$ is a prime such that $p^{2} \nmid N, \mathfrak{m}$ is a maximal ideal of $\mathbf{T}$ with residue characteristic $p$ such that if $p \mid N$, then $I_{f} \subseteq \mathfrak{m}$ for some newform $f$. We wish to show that then $\mathbf{T}$ and $\mathbf{T}^{\prime}$ agree locally at $\mathfrak{m}$.

If $p \nmid N$, then the result follows from Lemmas 5.11 and 5.20. If $f$ is a newform and $p \mid N$, then $U_{p}$ acts as \pm 1 on $f$, and hence $U_{p} \pm 1 \in I_{f}$. Thus if $p \mid N$ and $I_{f} \subseteq \mathfrak{m}$ for some newform $f$, then $U_{p}$ acts as a non-zero scalar $( \pm 1)$ on $H^{0}\left(X_{0}(N)_{\mathbf{F}_{p}}, \Omega_{X_{0}(N) / \mathbf{F}_{p}}\right)[\mathfrak{m}]$ (note that the action of $U_{p}$ on regular differentials was defined compatibly with the usual action of $U_{p}$ on complex differentials, i.e., on cuspforms; cf. the proof of Claim 1 in the proof of Lemma 5.20). The proposition follows again from Lemmas 5.11 and 5.20.

\section{Duality theory: AN APPENDIX By BRIAN CONRAD}

Let $k$ be a field and let $C$ be a proper reduced $k$-scheme with pure dimension 1 . Assume that $C$ is generically smooth, and let $C^{\prime} \subseteq C$ be a non-empty reduced closed subscheme with pure dimension 1 (so $C^{\prime}$ is also generically smooth). The case of most interest to us is when $C$ is a geometrically connected and semistable curve and $C^{\prime}$ is a smooth geometrically irreducible component. The inclusion $C^{\prime} \rightarrow C$ induces a natural map of $k$-groups $\mathrm{Pic}_{C / k} \rightarrow \mathrm{Pic}_{C^{\prime} / k}$, and on tangent spaces at the identity this is the canonical pullback map

$$
\theta: \mathrm{H}^{1}\left(C, \mathcal{O}_{C}\right) \rightarrow \mathrm{H}^{1}\left(C^{\prime}, \mathcal{O}_{C^{\prime}}\right)
$$

(as we see by computing with dual numbers over $k$ ). Each of $C$ and $C^{\prime}$ satisfies Serre's condition $\left(\mathrm{S}_{1}\right)$ by reducedness, so each is Cohen-Macaulay. Thus, by Serre duality we can identify the map of cotangent spaces with the map $\mathrm{H}^{0}\left(C^{\prime}, \omega_{C^{\prime} / k}\right) \rightarrow \mathrm{H}^{0}\left(C, \omega_{C / k}\right)$ dual to $\theta$. We wish to give a concrete description of this latter map. To do this, we first review some basic definitions and identifications in duality theory.

In what follows we use Grothendieck's approach to duality theory, which has the merit of permitting more localization operations than in Serre's approach. Since $C$ and $C^{\prime}$ are Cohen-Macaulay with pure dimension 1, their relative dualizing complexes over $k$ are naturally identified with $\omega_{C / k}[1]$ and $\omega_{C^{\prime} / k}[1]$ respectively [Con00, 3.5.1]. Since (by construction) the formation of the relative dualizing complex is compatible with Zariski-localization on the source, we have canonical isomorphisms $\left.\omega_{C^{\prime} / k}\right|_{C^{\prime s m}} \simeq \Omega_{C^{\prime s m} / k}^{1}$ and $\left.\omega_{C / k}\right|_{C^{s m}} \simeq \Omega_{C^{s m} / k}^{1}$ that coincide on the open locus $U=C^{\mathrm{sm}} \cap C^{\prime}$ that is dense in $C^{\prime}$ (and supported in $C^{\prime \text { sm }}$ ). If we let $j: C^{\mathrm{sm}} \rightarrow C$ and $j^{\prime}: C^{\prime \mathrm{sm}} \rightarrow C^{\prime}$ denote the canonical dense open immersions then by $[\mathrm{Con} 00,5.2 .1]$ the natural maps

$$
\omega_{C^{\prime} / k} \rightarrow j_{*}^{\prime}\left(\Omega_{C^{\prime s m} / k}^{1}\right), \omega_{C / k} \rightarrow j_{*}\left(\Omega_{C^{s m} / k}^{1}\right)
$$

are injective. By construction this is compatible with the natural isomorphism $\left.\left.\omega_{C / k}\right|_{U} \simeq \omega_{C^{\prime} / k}\right|_{U}$. Letting $\eta: \operatorname{Spec}(K) \rightarrow C$ and $\eta^{\prime}: \operatorname{Spec}\left(K^{\prime}\right) \rightarrow C^{\prime}$ denote 
the canonical maps from the schemes of generic points, $\omega_{C^{\prime} / k}$ maps isomorphically onto a coherent subsheaf of $\eta_{*}^{\prime}\left(\Omega_{K^{\prime} / k}^{1}\right)$ and likewise for $\omega_{C / k}$ in $\eta_{*}\left(\Omega_{K / k}^{1}\right)$; these image subsheaves are the so-called sheaves of regular differentials, and a classical result of Rosenlicht describes these images explicitly using residues when $k$ is algebraically closed [Con00, 5.2.3]. We will not require Rosenlicht's result for the statement or proof of the theorem below.

Using Grothendieck's theory of relative trace maps, the canonical closed immersion $\iota: C^{\prime} \rightarrow C$ over $k$ induces a trace morphism $\operatorname{Tr}_{\iota}: \iota_{*}\left(\omega_{C^{\prime} / k}\right) \rightarrow \omega_{C / k}$ whose formation commutes with Zariski-localization on $C$, so over the dense open $U=\iota^{-1}\left(C^{\mathrm{sm}}\right) \subseteq C^{\prime}$ it induces the natural isomorphism $\left.\omega_{C^{\prime} / k}\right|_{U} \simeq$ $\left.\omega_{C / k}\right|_{U}$, or equivalently it is the identity map on $\Omega_{U / k}^{1}$. Hence, $\operatorname{Tr}_{\iota}$ is compatible with the canonical inclusions $\omega_{C^{\prime} / k} \hookrightarrow \eta_{*}^{\prime}\left(\Omega_{K^{\prime} / k}^{1}\right)$ and $\omega_{C / k} \hookrightarrow \eta_{*}\left(\Omega_{K / k}^{1}\right)$. In particular, the map $\operatorname{Tr}_{\iota}$ is compatible with the natural identification of meromorphic 1-forms on $C^{\prime}$ with meromorphic 1-forms on $C$ (i.e., compatible with the injection $\Omega_{K^{\prime} / k}^{1} \hookrightarrow \Omega_{K / k}^{1}$ ).

Having summarized some inputs from duality theory, we can now state the result we want to prove.

TheOREM 6.1. The pullback $\mathrm{H}^{1}\left(C, \mathcal{O}_{C}\right) \rightarrow \mathrm{H}^{1}\left(C^{\prime}, \mathcal{O}_{C^{\prime}}\right)$ is dual to the natural map

$$
\mathrm{H}^{0}\left(C^{\prime}, \omega_{C^{\prime} / k}\right)=\mathrm{H}^{0}\left(C, \iota_{*}\left(\omega_{C^{\prime} / k}\right)\right) \rightarrow \mathrm{H}^{0}\left(C, \omega_{C / k}\right) .
$$

Proof. Let $\operatorname{Tr}_{C}: \mathrm{H}^{1}\left(C, \omega_{C / k}\right) \rightarrow k$ and $\operatorname{Tr}_{C^{\prime}}: \mathrm{H}^{1}\left(C^{\prime}, \omega_{C^{\prime} / k}\right) \rightarrow k$ be the canonical trace maps, so our problem is to prove that for $s \in \mathrm{H}^{1}\left(C, \mathcal{O}_{C}\right)$ and $\xi^{\prime} \in \mathrm{H}^{0}\left(C^{\prime}, \omega_{C^{\prime} / k}\right) \subseteq \Omega_{K^{\prime} / k}^{1}$,

$$
\operatorname{Tr}_{C^{\prime}}\left(\left.\xi^{\prime} \cup s\right|_{C^{\prime}}\right)=\operatorname{Tr}_{C}\left(\operatorname{Tr}_{\iota}\left(\xi^{\prime}\right) \cup s\right)
$$

in $k$. By the functoriality of Grothendieck's trace map, $\operatorname{Tr}_{C^{\prime}}=\operatorname{Tr}_{C} \circ \mathrm{H}^{1}\left(\operatorname{Tr}_{\iota}\right)$ as maps $\mathrm{H}^{1}\left(C^{\prime}, \omega_{C^{\prime} / k}\right) \rightarrow k$. Thus, it suffices to show that the map $\mathrm{H}^{1}\left(C^{\prime}, \omega_{C^{\prime} / k}\right) \rightarrow \mathrm{H}^{1}\left(C, \omega_{C / k}\right)$ induced by $\operatorname{Tr}_{\iota}$ carries $\left.\xi^{\prime} \cup s\right|_{C^{\prime}}$ to $\operatorname{Tr}_{\iota}\left(\xi^{\prime}\right) \cup s$. We may view dualizing sheaves as subsheaves $\omega_{C / k} \subseteq \eta_{*}\left(\Omega_{K / k}^{1}\right)$ and $\omega_{C^{\prime} / k} \subseteq$ $\eta_{*}^{\prime}\left(\Omega_{K^{\prime} / k}^{1}\right)$ in terms of which we have seen that the abstract trace map $\operatorname{Tr}_{\iota}$ is induced by the natural inclusion $\Omega_{K^{\prime} / k}^{1} \subseteq \Omega_{K / k}^{1}$.

To do the computation we work with Cech theory. Let $\left\{U_{n}\right\}$ be an ordered finite open affine cover of $C$ and let $U_{n}^{\prime}=U_{n} \cap C^{\prime}$, so $\left\{U_{n}^{\prime}\right\}$ is an open affine cover of $C^{\prime}$. The cohomology class $s$ corresponds to a Cech 1-cocycle $\left\{s_{n, m}\right\}_{n<m}$ with $s_{n, m} \in \mathcal{O}_{C}\left(U_{n} \cap U_{m}\right)$, so $s^{\prime}$ corresponds to $\left\{s_{n, m}^{\prime}\right\}$ with $s_{n, m}^{\prime}=\left.s_{n, m}\right|_{U_{n}^{\prime} \cap U_{m}^{\prime}}$. Identifying $\xi^{\prime}$ with an element of $\Omega_{K^{\prime} / k}^{1},\left.\xi^{\prime} \cup s\right|_{C^{\prime}} \in \mathrm{H}^{1}\left(C^{\prime}, \omega_{C^{\prime} / k}\right)$ corresponds to $\left\{s_{n, m}^{\prime} \xi^{\prime}\right\}_{n<m}$ and $\operatorname{Tr}_{\iota}\left(\xi^{\prime}\right) \cup s \in \mathrm{H}^{1}\left(C, \omega_{C / k}\right)$ corresponds to $\left\{s_{n, m} \xi^{\prime}\right\}_{n<m}$, where $\xi^{\prime}$ is viewed in $\Omega_{K / k}^{1}$ in the natural way. The product $s_{n, m} \xi^{\prime}$ at the generic points of $U_{n} \cap U_{m}$ vanishes at generic points not in $C^{\prime}$, so the required equality is clear even at the level of Cech 1-cocycles. 


\section{REFERENCES}

[AK70] Allen Altman and Steven Kleiman, Introduction to Grothendieck duality theory, Lecture Notes in Mathematics, Vol. 146, SpringerVerlag, Berlin, 1970.

[ARS06] A. Agashe, K. Ribet and W. Stein, The Manin Constant, Pure and Applied Mathematics Quarterly, Special issue: In honor of John H. Coates (2006), to appear.

[AS05] A. Agashe and W.A. Stein, Visible evidence for the Birch and Swinnerton-Dyer conjecture for modular abelian varieties of analytic rank zero, Math. Comp. 74 (2005), no. 249, 455-484, with an appendix by J. Cremona and B. Mazur.

[AU96] A. Abbes and E. Ullmo, À propos de la conjecture de Manin pour les courbes elliptiques modulaires, Compositio Math. 103 (1996), no. 3, 269-286.

[BCP97] W. Bosma, J. Cannon, and C. Playoust, The Magma algebra system. I. The user language, J. Symbolic Comput. 24 (1997), no. 3-4, 235265, Computational algebra and number theory (London, 1993).

[BCDT01] C. Breuil, B. Conrad, F. Diamond, and R. Taylor, On the modularity of elliptic curves over $\mathbf{Q}$ : wild 3-adic exercises, J. Amer. Math. Soc. 14 (2001), no. 4, 843-939 (electronic).

[BLR90] S. Bosch, W. Lütkebohmert, and M. Raynaud, Néron models, Springer-Verlag, Berlin, 1990.

[Con00] Brian Conrad, Grothendieck duality and base change, Lecture Notes in Mathematics, vol. 1750, Springer-Verlag, Berlin, 2000.

[Con07] Brian Conrad, Arithmetic moduli of generalized elliptic curves, J. Inst. Math. Jussieu 6 (2007), no. 2, 209-278.

[CK04] A. C. Cojocaru and E. Kani, The modular degree and the congruence number of a weight 2 cusp form, Acta Arith. 114 (2004), no. 2, 159167.

[Cre97] J.E. Cremona, Algorithms for modular elliptic curves, second ed., Cambridge University Press, Cambridge, 1997, available at http://www.maths.nott.ac.uk/personal/jec/book/.

[DI95] F. Diamond and J. Im, Modular forms and modular curves, Seminar on Fermat's Last Theorem, Providence, RI, 1995, pp. 39-133.

[Dia97] F. Diamond, The Taylor-Wiles construction and multiplicity one, Invent. Math. 128 (1997), no. 2, 379-391. 
[DR73] P. Deligne and M. Rapoport, Les schémas de modules de courbes elliptiques, Modular functions of one variable, II (Proc. Internat. Summer School, Univ. Antwerp, Antwerp, 1972) (Berlin), Springer, 1973, pp. 143-316. Lecture Notes in Math., Vol. 349.

[Fre97] G. Frey, On ternary equations of Fermat type and relations with elliptic curves, Modular forms and Fermat's last theorem (Boston, MA, 1995), Springer, New York, 1997, pp. 527-548.

[FM99] G. Frey and M. Müller, Arithmetic of modular curves and applications, Algorithmic algebra and number theory (Heidelberg, 1997), Springer, Berlin, 1999, pp. 11-48.

[Kil02] L.J.P. Kilford, Some non-Gorenstein Hecke algebras attached to spaces of modular forms, J. Number Theory 97 (2002), no. 1, 157164.

[KM85] N.M. Katz and B. Mazur, Arithmetic moduli of elliptic curves, Princeton University Press, Princeton, N.J., 1985.

[KW08] L. J. P. Kilford and Gabor Wiese, On the failure of the Gorenstein property for Hecke algebras of prime weight, Experiment. Math. 17 (2008), no. 1, 37-52. MR MR2410114 (2009c:11075)

[Li75] W-C. Li, Newforms and functional equations, Math. Ann. 212 (1975), 285-315.

[Maz77] B. Mazur, Modular curves and the Eisenstein ideal, Inst. Hautes Études Sci. Publ. Math. (1977), no. 47, 33-186 (1978).

[Maz78] B. Mazur, Rational isogenies of prime degree (with an appendix by D. Goldfeld), Invent. Math. 44 (1978), no. 2, 129-162.

[MR91] B. Mazur and K. A. Ribet, Two-dimensional representations in the arithmetic of modular curves, Astérisque (1991), no. 196-197, 6, 215-255 (1992), Courbes modulaires et courbes de Shimura (Orsay, $1987 / 1988)$.

[Mur99] M. R. Murty, Bounds for congruence primes, Automorphic forms, automorphic representations, and arithmetic (Fort Worth, TX, 1996), Amer. Math. Soc., Providence, RI, 1999, pp. 177-192.

[Rib75] K. A. Ribet, Endomorphisms of semi-stable abelian varieties over number fields, Ann. Math. (2) 101 (1975), 555-562.

[Rib81] K. A. Ribet, Endomorphism algebras of abelian varieties attached to newforms of weight 2, Seminar on Number Theory, Paris 1979-80, Progr. Math., vol. 12, Birkhäuser Boston, Mass., 1981, pp. 263-276. 
[Rib83] K. A. Ribet, Mod p Hecke operators and congruences between modular forms, Invent. Math. 71 (1983), no. 1, 193-205.

[Rib90] K. A. Ribet, On modular representations of $\mathrm{Gal}(\overline{\mathbf{Q}} / \mathbf{Q})$ arising from modular forms, Invent. Math. 100 (1990), no. 2, 431-476.

[RS01] Kenneth A. Ribet and William A. Stein, Lectures on Serre's conjectures, Arithmetic algebraic geometry (Park City, UT, 1999), IAS/Park City Math. Ser., vol. 9, Amer. Math. Soc., Providence, RI, 2001, pp. 143-232.

[Ser88] Jean-Pierre Serre, Algebraic groups and class fields, Graduate Texts in Mathematics, vol. 117, Springer-Verlag, New York, 1988, Translated from the French.

[Shi94] G. Shimura, Introduction to the arithmetic theory of automorphic functions, Princeton University Press, Princeton, NJ, 1994, Reprint of the 1971 original, Kan Memorial Lectures, 1.

[S+09] W. A. Stein et al., Sage Mathematics Software (Version 4.2), The Sage Development Team, 2009, http://www.sagemath.org.

[Stu87] J. Sturm, On the congruence of modular forms, Number theory (New York, 1984-1985), Springer, Berlin, 1987, pp. 275-280.

[Ti197] J. Tilouine, Hecke algebras and the Gorenstein property, Modular forms and Fermat's last theorem (Boston, MA, 1995), Springer, New York, 1997, pp. 327-342.

[Wie07] Gabor Wiese, Multiplicities of Galois representations of weight one, Algebra Number Theory 1 (2007), no. 1, 67-85, With an appendix by Niko Naumann.

[Wil80] Andrew Wiles, Modular curves and the class group of $\mathbf{Q}\left(\zeta_{p}\right)$, Invent. Math. 58 (1980), no. 1, 1-35.

[Wil95] A. J. Wiles, Modular elliptic curves and Fermat's last theorem, Ann. of Math. (2) 141 (1995), no. 3, 443-551.

[Zag85] D. Zagier, Modular parametrizations of elliptic curves, Canad. Math. Bull. 28 (1985), no. 3, 372-384. 